\title{
Research Square
The Rna-binding Protein GRSF1 Promote shepatocarcinogenesis via competitively Binding Toyy 1 mRNA with mIR-30e-5p
}

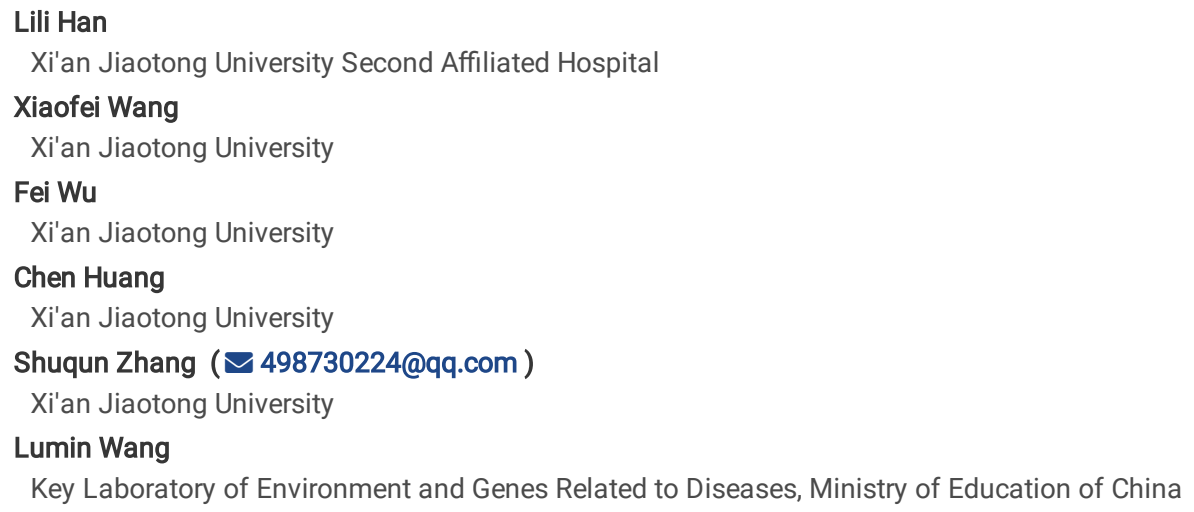

Research

Keywords: GRSF1, YY1, miR-30e-5p, RNA binding proteins, HCC, VE8

Posted Date: June 24th, 2020

DOI: https://doi.org/10.21203/rs.3.rs-35977/v1

License: (c) (1) This work is licensed under a Creative Commons Attribution 4.0 International License. Read Full License 


\section{Abstract}

Background:

Numerous studies have highlighted that dysregulation of RNA binding protein (RBP) expression is causally linked with human cancer tumorigenesis. The detailed biological effect and underlying mechanisms of RBP GRSF1 in hepatocellular carcinoma (HCC) remain unclear.

Methods:

GRSF1 expression in HCC tissues and cells was measured by western blotting and qRT-PCR assays. HCC cells with stable knockdown of GRSF1 were established using two sh-RNA encoding lentiviruses. Then the functions of GRSF1 in HCC were explored using MTT, colony formation, flow cytometry, Transwell assays and xenograft model. Transcriptometric sequencing in GRSF1-deficient MHCC-97H cells were carried out to identify the downstream effector of GSRF1. The regulatory mechanisms among GRSF1, YY1 and miR-30e-5p were investigated by RNA immunoprecipitation, luciferase assay, RNA pull down and ChIP assay.

Results:

GRSF1was frequently increased in HCC tissue and cells with a worse clinical outcomes in HCC. GRSF1 worked as a novel oncogenic RBP in vitro and in vivo via post-regulating the stability of YY1 mRNA. And the GUUU motifs on YY1 3

UTR2663-2847wasthespec if $i c b \in d \in$ gmot if $s f$ or GRSF1. M or e $\int$ erest $\in g$, YY1 feedbackpromotedGRSF1expressionbyb $\in d \in g$ UTR 2663-2847 region. At last, we identified that 3-amino-6-(4-methylsulfonylphenyl)-N-phenylpyrazine-2-carboxamide (VE821) blocked HCC progression by inhibiting the GRSF1/YY1 pathway.

Conclusions:

GRSF1 promoted hepatocarcinogenesis via competitively binding to YY1 3'UTR 2663-2847 with miR-30e-5p.The interaction network among GRSF1, YY1 and miR-30e-5p provided a new insight for the HCC pathogenesis, and VE821 may serve as a novel agent with potential for HCC treatment via inhibiting GRSF1/YY1 axis.

\section{Introduction}

Hepatocellular carcinoma (HCC) is one of the most commonly diagnosed cancer worldwide with poor prognosis ${ }^{[1,2]}$. Despite intensive efforts to improve effective monitoring and develop more therapeutic strategies, HCC patients still face unsatisfactory prognosis ${ }^{[3]}$. Therefore, exploring the pathogenesis of $\mathrm{HCC}$ and finding new effective therapeutic targets is of great concern.

It is recognized that RNA binding proteins (RBPs) are of immense importance in diverse biological regulatory processes. In the nucleus, RBPs regulate gene transcription, RNA splicing and RNA nuclear export ${ }^{[4-6]}$. In the cytoplasm, RBPs influence RNA degradation, stabilization, modification and translation ${ }^{[4-6]}$. Mounting evidence indicates that dysregulation of RBPs contribute to the transcriptomic imbalance and thus drive tumorigenicity [7-9]. G-rich sequence binding factor 1 (GRSF1), an RNA-binding protein identified recently, has a significant impact in almost all steps of post-transcriptional regulation by binding with the target mRNAs through its three RRM (RNA-binding) domains. GRSF1 is essential in preventing premature senescence induced by oxidative stress [10] Recent observation determined GRSF1 was implicated in cancer progression. It is reported that GRSF1 could drive the metastasis of cervical cancer cells via the PIK3R3/AKT/NF-KB and TIMP3/MMP9 pathways ${ }^{[11]}$. In addition, GRSF1 has been revealed to promote cervical cancer by enhancing TMED5 and LMNB1 expression ${ }^{[12]}$. However, far less is known about the potential function and underlying mechanism of GRSF1 in HCC. The data of this current study first discovered that GRSF1 is frequently increased in HCC and promotes hepatocarcinogenesis as an RBP.

Yin-Yang 1 (YY1), could act as a transcriptional activator of oncogenes or a repressor of cancer suppressor, is a critical promoter of hepatocarcinogenesis ${ }^{\text {[13- }}$

${ }^{15]}$. Extensive evidences confirm that YY1 is significantly increased in HCC tissue, closely correlates with numbers of cancer related genes and HCC tumorigenesis. However, the upstream regulatory mechanism of $Y Y 1$ in HCC remains uncharted. This study identified YY1 was an essential downstream effector of GRSF1, and overexpression of YY1 rescued tumor-inhibiting effect of GRSF1 knockdown, suggesting GRSF1 promote hepatocarcinogenesis via promotingYY1 expression in HCC.

MicroRNAs (miRNAs or miRs) are a group of non-coding RNAs which are 18-24 nucleotides in length. MicroRNAs are involved in regulating various biological processes in HCC ${ }^{[16-18]}$. MiR-30e-5p, a novel cancer-related miRNA, is involved in the progression of various human cancer. For example, it is elucidated that MiR-30e-5p inhibited nasopharyngeal carcinoma migration by directly repressing MAT1 ${ }^{[19]}$. And miR-30e-5p was reported to impair non-small cell lung

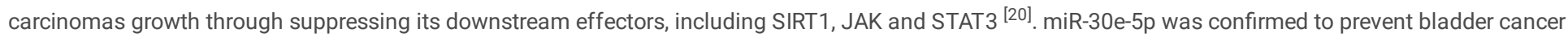
tumorigenesis by inhibiting MTDH ${ }^{[21]}$. However, the role of miR-30e-5p in HCC and its related pathway is less reported. In this study, we uncovered that miR30e-5p acted as a tumor suppressor in HCC via regulating YY1 as an upstream regulator competing with GRSF1.

In conclusion, we discovered RBP GRSF1 promoted HCC tumorigenesis in vitro and in vivo via enhancing YY1 stability, and the GUUU motifs on YY1 3 UTR2663-2847wasthespec if icb $\in d \in$ gmot if $s f$ or GRSF1. M or e $\int$ erest $\in$ g, ourdatashowedtŶY1activatedGRSF1 promoter and en Processing math: $14 \%$ 
UTR 2663-2847 region with miR-30e-5p. Finally, we identified that VE-821, a small molecular compound, blocked HCC progression by inhibiting the GRSF1/YY1 pathway, providing novel potential treatment option for HCC.

\section{Methods}

\section{Patient tissue specimens}

The ethics committee of Xi'an Jiaotong University approved this study (Approval number: 130043). A total of 120 HCC samples and paired noncancerous tissues were obtained from $\mathrm{HCC}$ patients during the resection of $\mathrm{HCC}$ lesions. All the patients diagnosed by two pathologists did not received radiotherapy, immunotherapy or chemotherapy before surgery.

\section{Quantitative real-time PCR.}

Total RNA was extracted from HCC cells or tissues using TRIzol (Invitrogen, Carlsbad, CA, USA) reagent followed the manufacturer's description. RNA samples was reversed into cDNA using TaKaRa Reverse Transcription System (TaKara, Dalian, China). qRT-PCR was performed using Takara SYBR Premix kit (Takara Bio, Inc.). The RNA level was expressed as a relative results via the $2^{-\triangle \triangle C T}$ method against GAPDH or U6, which was used as the endogenous standard control. The primer sequences used here are shown in Supplementary Table 1 (Table S1)

\section{Western blotting}

The protein was obtained with RIPA buffer (Beyotime, China) from HCC tissues or cells and quantified by a BCA protein assay kit (ThermoFisher, USA). Equal amounts $(25 \mu \mathrm{g})$ of protein sample were separated by SDS-PAGE and transferred to PVDF membranes (Millipore, Bellerica, MA), followed by blocking in $5 \%$ nonfat milk. After incubated in primary antibodies against GRSF1 (ab241400, Abcam), YY1 (\#63227, CST), or GAPDH (ab6922; Abcam) overnight at $4{ }^{\circ} \mathrm{C}$, the membranes were probed in the secondary antibodies at room temperature for $1 \sim 2 \mathrm{~h}$. The data was collected in an ECL blotting analysis system (Amersham Pharmacia Biotech, Piscataway, NJ).

\section{Cell lines and cells culture}

Human HCC cells HCC cell lines (Huh7, Hep3B, MHCC-97H, MHCC-97L and Bel-7402) and the immortalized normal human liver cell line (LO2) were bought from FuHeng Cell Shanghai Center (China), and maintained in Dulbecco's modified Eagle medium (DMEM, Sigma-Aldrich; Merck KGaA) with 10\% FBS(Biological Industries, CT, USA). All the cells used in this study were cultured at $37^{\circ} \mathrm{C}$ with $5 \% \mathrm{CO}_{2}$.

\section{Cells transfection}

The sh-RNA encoding lentiviruses against GRSF1 or YY1, including sh-GRSF1-1, sh-GRSF1-2, sh-YY1-1 and sh-YY1-2, and the lentiviruses vectors used to overexpress the GRSF1 or YY1, such as ov-GRSF1 and ov-YY1, as well as their negative control vector were all purchased from Genepharma (Shanghai, China). These lentiviruses vectors were transfected into $\mathrm{MHCC}-97 \mathrm{H}$ or Hep3B cells according to the manufacturer's instruction, when the confluence of cells in 6-well plates reached 50\%-60\%. The medium was replaced by fresh normal medium after $6 \mathrm{~h}$. After $48 \mathrm{~h}$, the transfection efficiency was assessed by qRT-PCR and western blotting assays.

\section{Cell proliferation assay}

MTT assay and colony-formation assay were performed to investigate HCC cells proliferation. For MTT assay, transfected HCC cells were added in 96-well plates at a density of 500 cells per well. At the indicated times points, MTT solution was treated into the plates. After incubated at room temperature for 4 hours, the medium was replaced by those containing dimethyl sulfoxide (DMSO). At last, the absorbance was measured under a plate reader (BioTek, USA). For colony-formation assay, HCC cells were added in 12-well plates according to 100 cells per well and normally cultured for 10 days. Then the cells were stained with $0.1 \%$ crystal violet, washed with PBS and then dried. The colony-forming units were measured under a microplate reader (Nikon, Tokyo, Japan).

\section{Flow cytometry}

The transfected cells were seeded in a 6-well plates $\left(3 \times 10^{5}\right.$ cells per well). After cultured for $24 \mathrm{~h}$, the cells were double-stained with Annexin-v buffer and PI solution. Finally, cells apoptosis was measured by BD LSRFortessa (BD Biosciences).

\section{Cell migration and invasion assays}

The $3 \times 10^{4}$ transfected cells in $200 \mu \mathrm{FBS}$ free media were added into the chamber of the Transwells (Millipore) hanged in 24-well plate. Normal culture media with $10 \%$ FBS was treated into the corresponding well. After $48 \mathrm{~h}$, cells left in the top chamber were removed, while cells successfully migrated into underside of the membrane were fixed with $4 \%$ paraformaldehyde and stained with $0.1 \%$ crystal violet. At last, the images were collected by microscope. For invasion assay, the membranes of chambers were coated with were precoated with Matrigel (BD Biosciences, San Jose, CA).

\section{RNA immunoprecipitation (RIP assay)}

MHCC-97H or Hep3B cells were transfected with ov-GRSF1 lentiviruses vectors as described previously. After $48 \mathrm{~h}, 1 \times 10^{7}$ transfected cells were mixed with RIP Lysis Buffer (Millipore, USA) supplemented with an RNase inhibitor and a protease inhibitor cocktail. A total of $4 \mu \mathrm{g}$ control rabbit IgG or anti-GRSF1 antibody was incubated with the protein-A Sepharose beads for $2 \mathrm{~h}$ at $4{ }^{\circ} \mathrm{C}$, then the mixture was added into cells lyse and incubated at $4{ }^{\circ} \mathrm{C}$ overnight. RNA was isolated and purified by phenol, chloroform and isoamyl alcohol. At last, qRT-PCR was conducted to evaluate YY1 transcripts.

\section{Biotinylated RNA pull-down assay}


The different PCR fragments of YY1 mRNA, including 5UTR, CDS, 3UTR-1348-2204, 2205-3060, 3061-3916, 3917-4772, 4773-5627 and 5628-6481, 3UTR 2205-2418, 2419-2662, 2663-2847(WT) and 2848-3060, as well as M1, M2 and M3 mutant of 3UTR 2663-2847 were used as templates for transcription. The biotin-CTP (Promega) and T7 polymerase (Promega) were used to prepare Biotinylated transcripts. The mixture of $1 \mu \mathrm{g}$ biotinylated transcripts, $40 \mu \mathrm{g}$ cell lysates and $10 \mathrm{~m}$ TENT buffer were incubated with continuous rotation at room temperature for $2 \mathrm{~h}$. The complexes were collected by paramagnetic streptavidin-conjugated Dynabeads (Invitrogen). And then western blotting was performed to measure the pull-down materials with an antibodies against GRSF1 and GAPDH.

\section{Luciferase reporter assay}

The sequence of different YY1 mRNA fragments, including 5UTR, CDS, 3UTR-1348-2204, 2205-3060, 3061-3916, 3917-4772, 4773-5627, 5628-6481, and 3'UTR 2205-2418, 2419-2662, 2663-2847, 2848-3060 was amplified and cloned into a luciferase reporter vector. The sh-GRSF1 or sh-Ctrl vectors, and luciferase reporter vectors were transfected into HCC cells according to the manufacturer's description. After $48 \mathrm{~h}$, luciferase activity was assessed with the Dual-Luciferase Assay kit (Promega, WI, USA).

\section{RNA stability measurement}

After treated with VE821 or sh-GRSF1/sh-Ctrl vectors, MHCC-97H or Hep3B cells were added with actinomycin D (5 mg/ml) to abolish de novo synthesis of RNA. Then the RNA extraction at indicated times and qRT-PCR was performed as described previously.

\section{Reporter constructs.}

Firstly, YY1 3UTR mutations in GRSF1-binding site (YFP-YY1-GRSF1 MT) or the miR-30e-5p-binding site (YFP-YY1-miR-30e-5p MT) were structured using Agilent Technologies site-directed mutagenesis kit (Palo Alto, CA, USA). Subsequently, YY1 3UTR (YFP-YY1 WT) and the mutations were amplified and then introduced into the pd2EYFP-N1 reporter (YFP) vector (Clontech, Palo Alto, CA, USA). The sequence used to be amplified are shown in Supplementary Table 2 (Table S2).

\section{Chromatin immunoprecipitation (ChIP)}

ChIP assay was carried out using a Enzymatic Chromatin IP Kit (Thermo Fisher Scientific, Rockford, IL) followed the protocol in the manufacturer. MHCC-97H and Hep3B cells were crosslinked using 1\% formaldehyde and then collected with ChIP lysis buffer. After digested with the micrococcal nuclease, immunoprecipitation samples were mixed with protein A/G magnetic beads (Millipore) and rotationally incubated with normal IgG or specific antibodies overnight at $4{ }^{\circ} \mathrm{C}$. After digested by Proteinase K, DNA were extracted using phenol, chloroform and isoamyl alcohol. Finally, the purified DNA was analyzed by qRT-PCR.

\section{Molecule compound}

Small molecule compound VE821 (C18H16N403S, molecular weight 368.4, CAS No.1232410-49-9) was obtained from the Department of Chemistry, Xi'an Jiaotong University. The high-performance liquid chromatography determined that the purity of VE821 was more than $97 \%$.

\section{Protein degradation analysis}

After treated with or without VE821 for $48 \mathrm{~h}, \mathrm{MHCC}-97 \mathrm{H}$ cells in different dish were added with cycloheximide (CHX, $50 \mathrm{~g} / \mathrm{ml})$ at the same time. At indicted time points $(0,5,10,15,20,25,30,35,40$ and $45 \mathrm{~h})$, protein samples were collected and then analyzed by western blotting assay.

\section{Animal model assays}

Aged 5 weeks male nude mice were purchased from Animal center of Xi'an Jiaotong University and feeded in a specific-pathogen-free environment. $1 \times 10^{7}$ indicated HCC cells in $150 \mu \mathrm{l}$ serum-free media were injected to the right flank of the mice. After tumor xenografts could be observed directly, the tumor sizes were measured using the formula length $\times$ width $2 \times 0.5$ every other day. For the two drug treatment groups, mice were received intraperitoneal injection of the Vehicle or VE821 (15 mg/kg) twice a week for 20 days. After 30 days, the mice were sacrificed, and the tumors in the xenografts models were surgically removed for further analysis.

\section{Immunohistochemistry (IHC)}

The IHC analysis was conducted as described in our previous report ${ }^{[7]}$. The primary antibodies against GRSF1, YY1, Ki67, as well as the secondary antibody were all obtained from Beijing Biosynthesis Biotechnology co., LTD.

\section{Statistical analysis}

All statistical analyses in this study were conducted with GraphPad Prism Software and R software (version 3.3.3), each experiment was repeated triplicate, and the results were expressed as a mean \pm SE. The Chi-square tests was used to identify the association between HCC clinicopathological feather and GRSF1. The Kaplan-Meier survival analysis with log-rank test was used to assess the correlation between outcome of HCC patients and GRSF1 expression. For comparisons the differences, Student's t-test was used between two groups, and One-way ANOVA was used among more than two groups. *p $\mathbb{0} 0.05$ with a two-sided test was considered statistically significant.

\section{Results}

GRSF1 is increased in HCC and associated with poor prognosis.

Analysis of public datasets (Cancer Genome Atlas, TCGA) suggested that the expression of GRSF1 was elevated in HCC tissues compared with normal tissues Processing math: 14\% Irvival analysis using data from TCGA revealed higher GRSF1 expression indicated a shorter overall survival (OS) for HCC patients 
(Fig. 1B, p < 0.01). To further investigate the expression pattern of GRSF1, we measured GRSF1 expression in 120 pairs of samples, including HCC tissues and adjacent nontumor clinical samples, and in HCC cell lines. The results of qRT-PCR assay demonstrated that GRSF1 was obviously higher in the HCC tissues (Fig. 1C, p < 0.01). In line with the test in clinical samples, qRT-PCR analysis and western blot assays suggested that both GRSF1 mRNA and protein expression levels were markedly increased in HCC cell lines compared with the immortalized hepatic cell line Lo2 (Fig. 1D, p<0.05, $p<0.01$ ). We further evaluated the expression of GRSF1 in HCC tissues and the matched no cancerous tissues using western blot assay. Figure 1E showed GRSF1 protein expression level was increased in HCC tissues in $10 \mathrm{HCC}$ patients. The results of Kaplan-Meier survival analysis revealed that in total of $120 \mathrm{HCC}$ patients, patients with higher GRSF1 tend to have a worse outcomes, while those with lower GRSF1 expression had better outcomes $(p<0.01, \mathrm{Fig} .1 \mathrm{~F})$.

Furthermore, Chi-square tests suggested that the upregulation of GRSF1 was positively correlated with larger tumor size $(p<0.01)$, worse differentiation $(p<$ $0.01)$, microscopic vascular invasion $(p=0.009)$ and advanced TNM stage in HCC $(p<0.01$, Table 1$)$. In short, the increased GRSF1 was observed in HCC tissues and cell lines and associated with an advanced stage of carcinogenesis, as well as with a worse patient prognosis.

Table 1

Association between GRSF1 expressions and clinicopathological features in HCC

\begin{tabular}{|c|c|c|c|c|}
\hline \multirow[t]{2}{*}{ Variable } & \multirow[t]{2}{*}{ Total no. of patients $n=120$} & \multicolumn{2}{|l|}{ GRSF1 } & \multirow[t]{2}{*}{$\mathbf{p}$} \\
\hline & & $\begin{array}{l}\text { Low } \\
\text { expression }\end{array}$ & $\begin{array}{l}\text { High } \\
\text { expression }\end{array}$ & \\
\hline Age (years) & & & & 0.44 \\
\hline$<49$ & $57(47.5 \%)$ & 16 & 41 & \\
\hline$\geq 49$ & $63(52.5 \%)$ & 26 & 37 & \\
\hline \multicolumn{5}{|l|}{ Gender } \\
\hline Female & $12(10 \%)$ & 1 & 11 & 0.093 \\
\hline Male & $108(90 \%)$ & 41 & 67 & \\
\hline $\operatorname{AFP}(\mathrm{ng} / \mathrm{mL})$ & & & & $<0.031^{*}$ \\
\hline$<400$ & $69(57.5 \%)$ & 29 & 40 & \\
\hline$\geq 400$ & $51(42.5 \%)$ & 13 & 38 & \\
\hline Tumor size (cm) & & & & $<0.01 * *$ \\
\hline$<5$ & $51(42.5 \%)$ & 25 & 26 & \\
\hline$\geq 5$ & $69(57.5 \%)$ & 17 & 52 & \\
\hline Tumor multiplicity & & & & 0.099 \\
\hline Single & $79(65.83 \%)$ & 33 & 46 & \\
\hline Multiple & $41(34.17 \%)$ & 9 & 32 & \\
\hline $\begin{array}{l}\text { Differentiation } \\
\text { (Edmondson-Steiner) }\end{array}$ & & & & $<0.01 * *$ \\
\hline$|-| \mid$ & $59(49.17 \%)$ & 26 & 33 & \\
\hline III-IV & $61(50.83 \%)$ & 16 & 45 & \\
\hline Microscopic vascular invasion & & & & $0.009 * \star$ \\
\hline No & $71(59.17 \%)$ & 32 & 39 & \\
\hline Yes & $49(40.83 \%)$ & 10 & 39 & \\
\hline Stage & & & & 0.001 ** \\
\hline HII & $71(59.17 \%)$ & 30 & 41 & \\
\hline III-IV & $49(40.83 \%)$ & 12 & 37 & \\
\hline
\end{tabular}


Table 1

Association between GBP2 expressions and clinicopathological features in HCC

\begin{tabular}{|c|c|c|c|c|}
\hline \multirow[t]{2}{*}{ Variable } & \multirow[t]{2}{*}{ Total no. of patients $n=120$} & \multicolumn{2}{|l|}{ GBP2 } & \multirow[t]{2}{*}{$p$} \\
\hline & & $\begin{array}{l}\text { Low } \\
\text { expression }\end{array}$ & $\begin{array}{l}\text { High } \\
\text { expression }\end{array}$ & \\
\hline Age (years) & & & & 0.08 \\
\hline$<49$ & $57(47.5 \%)$ & 5 & 52 & \\
\hline$\geq 49$ & $63(52.5 \%)$ & 36 & 27 & \\
\hline \multicolumn{5}{|l|}{ Gender } \\
\hline Female & $12(10 \%)$ & 1 & 11 & 0.099 \\
\hline Male & 108(90\%) & 40 & 68 & \\
\hline $\operatorname{AFP}(\mathrm{ng} / \mathrm{mL})$ & & & & 0.081 \\
\hline$<400$ & $69(57.5 \%)$ & 35 & 34 & \\
\hline$\geq 400$ & $51(42.5 \%)$ & 6 & 45 & \\
\hline Tumor size $(\mathrm{cm})$ & & & & $0.045^{\star}$ \\
\hline$<5$ & $51(42.5 \%)$ & 7 & 44 & \\
\hline$\geq 5$ & $69(57.5 \%)$ & 34 & 35 & \\
\hline Tumor multiplicity & & & & $0.040 *$ \\
\hline Single & $79(65.83 \%)$ & 31 & 48 & \\
\hline Multiple & $41(34.17 \%)$ & 10 & 31 & \\
\hline $\begin{array}{l}\text { Differentiation } \\
\text { (Edmondson-Steiner) }\end{array}$ & & & & 0.001 ** \\
\hline$|-| \mid$ & $59(49.17 \%)$ & 10 & 49 & \\
\hline III-IV & $61(50.83 \%)$ & 31 & 30 & \\
\hline Microscopic vascular invasion & & & & $0.000 * *$ \\
\hline No & $71(59.17 \%)$ & 28 & 43 & \\
\hline Yes & $49(40.83 \%)$ & 13 & 36 & \\
\hline Stage & & & & $0.000 * *$ \\
\hline HII & $71(59.17 \%)$ & 28 & 43 & \\
\hline III-IV & $49(40.83 \%)$ & 13 & 36 & \\
\hline${ }^{\star} p<0.05 .{ }^{* *} p<0.01$ & & & & \\
\hline
\end{tabular}

\section{GRSF1 promoted HCC cell proliferation, invasion and migration}

The stably silenced GRSF1 in MHCC-97H and Hep3B cells were established using two different effective sh-RNA encoding lentiviruses (sh-GRSF1-1 and shGRSF1-2). Figure 2A showed the knockdown efficiency was confirmed by qRT-PCR and western blotting assay $(p<0.05$, Fig. $2 A)$. Functionally, the results of MTT assay uncovered that the proliferation ability of MHCC-97H and Hep3B cells were reduced following GRSF1 downregulation ( $p<0.05$, Fig. 2B).In addition, colony-forming ability of MHCC-97H and Hep3B cells was inhibited with the transfection of sh-GRSF1-1 or sh-GRSF1-1 vectors ( $p<0.01$, Fig. 2C). The flow cytometry (FCM) assay suggested that GRSF1 knockdown increased HCC cells apoptosis $(p<0.05$, Fig. 2D). Transwell assays demonstrated that GRSF1 knockdown repressed migration and invasion of MHCC-97H and Hep3B cells ( $p<0.01$, Fig. 2E). These data revealed that GRSF1 promoted HCC malignant biological behavior in vitro.

\section{GRSF1 promoted YY1 expression by directly binding to YY1 3`UTR}

To better understand the mechanism of its tumorigenic function, transcriptometric sequencing was performed on MHCC-97H GRSF1- deficient cells (shGRSF1) or control cells (sh-Ctrl). Since our results has uncovered GRSF1 promoted HCC cells proliferation, invasion and migration. Hence, we pay more attention to the downstream genes that can extensively regulate HCC phenotypes. YY1, which is pivotal to promote hepatocarcinogenesis via a wide variety of classical pathways, including MAPK, PI3K/AKT and Wnt/ $\beta$-catenin, was here identified as one of the genes with the most dramatic changes (fold-change > 2) with the downregulation of GRSF1 (Fig. 3A). Thus, we focused on YY1 as downstream effector of GRSF1. We performed qRT-PCR assays using 120 HCC clinical samples, the results confirmed that YY1 expression was markedly higher in HCC tissues than in normal tissues ( $p<0.01$, Fig. 3B). qRT-PCR assay Processing math: 14\% g GRSF1 failed to downregulate YY1 mRNA expression (Fig. 3C). However, protein levels of YY1 were significantly decreased with the 
knockdown of GRSF1 in MHCC-97H and Hep3B cells ( $p<0.01$, Fig. 3D). To further confirm the regulation of GRSF1 on YY1 in HCC, we established stably overexpressed GRSF1 in MHCC-97H and Hep3B cells via lentivirus approach (ov-GRSF1). Similarly, overexpression of GRSF1 could not upregulate YY1 mRNA expression (Fig. S1A). However, YY1 protein expression were significantly increased followed the GRSF1 overexpression in MHCC-97H and Hep3B cells ( $\mathrm{p}<$ 0.01 , Fig. S1B). These data suggesting that the correlation between GRSF1 and YY1 maybe upon a post-transcriptional pathway.

Mechanistically, RIP assays uncovered YY1 mRNA was enriched in GRSF1 IP antibody precipitated sample instead of IgG-IP sample, strongly suggesting that GRSF1 directly interacted with YY1 mRNA in HCC (Fig. 3E). Given that RBPs usually governed target genes expression and function via modulating the length of half-life of their mRNA, we further evaluated the stability of YY1 mRNA in response to GRSF1 knockdown in HCC cells. The results demonstrated that the half-life of YY1 mRNA was markedly shorter in GRSF1- deficient HCC cells than those of the corresponding control cells (Fig. 3F).

To investigate the specific binding region of GRSF1 on YY1 mRNA, we separated the full length of the YY1 3UTR into six overlapping fragments, including YY1 3UTR-1348-2204, 2205-3060, 3061-3916, 3917-4772, 4773-5627 and 5628-6481 (Fig. 3G) followed with performed pull-down assays. As shown in Fig. 3H, GRSF1 specifically interacted with 3UTR-2205 (nt 2205-3060), but not the 5UTR, CDS or other 3UTR fragments. Subsequently, we subdivided 3UTR2205 into four parts fragments (3UTR 2205-2418, 2419-2662, 2663-2847 and 2848-3060) and found that GRSF1 predominantly interacted with 3UTR 2663-2847 region instead of other fragments (Fig. 3I). Luciferase reporter assays in 293T cells were also carried out. Indeed, the knockdown of GRSF1 only decreased the luciferase activity of YY1 3UTR but not of YY1-5UTR or YY1-CDS (Fig. 3J). YY1 3UTR was then divided into six parts as previously mentioned, including YY1 3UTR-1348-2204, 2205-3060, 3061-3916, 3917-4772, 4773-5627 and 5628-6481 and generated the corresponding luciferase reporter plasmids. The results showed RGSF1 specifically interacted with 3'UTR-2205 (nt 2205-3060), which had been validated to bind to GRSF1 protein, whereas the luciferase activity of other fragments was not changed (Fig. 3J). We further subdivided 3UTR-2205 into four parts fragments (3UTR 2205-2418, 24192662, 2663-2847 and 2848-3060). Using the same method, we observed that YY1 3UTR 2663-2847 sharply lost luciferase activity upon GRSF1 knockdown. Altogether, these findings demonstrated that GRSF1 promoted YY1 expression via stabilizing YY1 mRNA by directly binding with its 3UTR $2663-2847$.

\section{YY1 worked as an essential downstream effector of GRSF1 and feedback promoted GRSF1 expression by binding to GRSF1 promoters}

To identify whether YY1 is essential for maintaining the function of GRSF1 in HCC, we performed a rescue assay. YY1 was stably overexpressed in GRSF1deficient MHCC-97H and Hep3B cells though a virus transfection pathway $(p<0.01$, Fig. 4A). From a functional point of view, YY1 overexpression rescued tumor-inhibiting effect of decreased GRSF1 on proliferation $(p<0.05$, Fig. 4B), colony formation $(p<0.01$, Fig. $5 \mathrm{C})$, cell apoptosis $(p<0.05$, Fig. 4D), invasion and migration $(p<0.01$, Fig. 4E) ability of MHCC-97H and Hep3B cells.

In the rescue assay, we unexpectedly found that GRSF1 expression in GRSF1-deficient MHCC-97H and Hep3B cells were concomitantly up-regulated with increased YY1 ( $p<0.01$, Fig. 4A). Thus, we speculated that YY1 was not only a downstream target of GRSF1, but also a feedback promoter of GRSF. Using the UCSC Genome Browser on Human feb.2009 (GRCh37/hg19) assembly software, we found that there was a potential binding site of YY1 in the promoter of GRSF1. We structured MHCC-97H and Hep3B cells with stably silenced or overexpressed YY1 using lentiviruses vectors, and found GRSF1 mRNA expression was markedly decreased or increased with upon the change of YY1 expression ( $p<0.01$, Fig. 4F). The protein expression level of GRSF1 showed the same changes $(p<0.01$, Fig. $4 \mathrm{G})$. Mechanistically, ChIP assays demonstrated that YY1 bound to the promoter of GRSF1, but not to the control region ( $p<0.01$, Fig. $4 \mathrm{H})$.

\section{miR-30e-5p inhibited YY1 and hepatocarcinogenesis via binding with 3'UTR of YY1}

The upstream regulation network of YY1 is very complex and not very clear. We hope to further explore the role of GRSF1 in the upstream regulation network of $Y Y 1$. Previous reports reveal $Y Y 1$ is regulated by microRNAs, thus we try to clarify whether there is competition between GRSF1 and microRNAs in the regulation process of $\mathrm{YY} 1$.

We accessed the public database, TargetScan (http://www.targetscan.org/) to search for miRNAs that could potentially interact with YY1 3UTR 2663-2847, and found that the complementary sequence of miR-30e-5p was contained in the YY1 3UTR 2663-2847 region (Fig. 5A). The role of miR-30e-5p in HCC has been rarely reported, therefore we carried out qRT-PCR assay to evaluate the expression of miR-30e-5p in 120 clinical HCC samples. The results showed that the miR-30e-5p expression was lower in the HCC tissues than those in the non-cancerous tissues $(p<0.01$, Fig. 5B). Transfection of precursor miR-30e-5p (premiR-30e-5p) markedly increased miR-30e-5p expression in MHCC-97H and Hep3B cells $(p<0.01$, Fig. $5 \mathrm{C})$. Luciferase reporter gene assay showed the increased miR-30e-5p reduced the luciferase activity of $Y Y 1$ with a wt $Y Y 1$ 3'-UTR, demonstrating that miR-30e-5p directly bound with $Y Y 1$ ( $p<0.01$, Fig. 5D). qRT-PCR assay and western blot assays confirmed $Y Y 1$ mRNA and protein expression levels were downregulated upon the transfection with pre-miR-30e-5p ( $p$ $<0.01$, Fig. 5E). Subsequently, we upregulated YY1 expression in the miR-30e-5p-overexpressing HCC cells though a virus transfection pathway (Fig. 5E). Functionally, colony formation assay, transwell assays and MTT assay suggested that increased miR-30e-5p suppressed HCC cells colon forming, migration, invasion and proliferation, while YY1 overexpression counteracted the tumor-inhibiting effect induced by pre-miR-30e-5p ( $p<0.01$, Fig. 5F-H). These findings demonstrate that miR-30e-5p acted as a tumor suppressor via directly inhibiting YY1, and YY1 could counteracted the functional effects of miR-30e-5p in hepatocarcinogenesis.

\section{GRSF1 and miR-30e-5p competitively regulated YY1 via binding to its 3'UTR}

Given that GRSF1 directly bound with YY1 3UTR 2663-2847, we try to identify the essential motifs which interacted with RNA-binding domains of GRSF1 in this region. We found that the region of 3UTR 2663-2847 contains three GUUU motifs that appear in the 3'UTR of YY1 mRNA frequently. Thus, mutants were structured, including M1 mutated in first GUUU motifs (GUUU to UGUG), M2 mutated in second GUUU motifs, M3 mutated in third GUUU motifs and M4 in which all the three GUUU motifs were replaced by UGUG (Fig. 6A). Subsequently, the biotin-mediated RNA pull-down assay was performed to measure the function of the wild-type fragment (WT) and four mutants on interacting with GRSF1.The results showed that the M1, M2 or M3 mutant lost partial capacity to 
associate with GRSF1, while the M4 mutant basically lost all the ability to interact with GRSF1 (Fig. 6B), suggesting the GUUU motifs on YY1 3'UTR 26632847 are GRSF1 binding motifs.

The binding site of YY1 for miR-30e-5p is partially overlapped with GRSF1-binding sites (Fig. 6C), and our results have shown that miR-30e-5p and GRSF1 play a hostile role in regulatory of YY1. Thus, we prepared the YFP reporter expressed a chimeric RNA construct of the sequence of YFP, wild-type (YFP-YY1 WT) or mutated YY1 3UTR to help better understand the co-regulation of YY1 mRNA by GRSF1 and miR-30e-5p. The construct only containing YFP sequence was identified as YFP Ctrl. While YFP-YY1-miR-30e-5p MT contained mutations within binding site of YY1 for miR-30e-5p. YFP-YY1-GRSF1 MT contained mutations that replaced GUUU motifs on YY1 3UTR 2663-2847 with UGUG (Fig. 6C).

After the transfection of sh-GRSF1 vector into MHCC-97H cells for $24 \mathrm{~h}$, YFP reports were treated in and then RIP assays confirmed that miR-30e-5p bound to YY1 3'UTR 2663-2847 region, the binding function was impaired by the transfection of YFP-YY1-miR-30e-5p MT (Fig. 6D).The mutation induced by the transfection of YFP-YY1-GRSF1 MT enhanced the interaction between miR-30e-5p and YY1 instead of disrupted their association, suggesting GRSF1 could antagonize miR-30e-5p binding with YY1 and the mutation of GRSF1 could not prevent miR-30e-5p binding to YY1 3`UTR (Fig. 6D). Thus, our data showed that GRSF1 and miR-30e-5p competitively regulate YY1 expression because of their binding sites overlap.

We further measure the function of GRSF1 knockdown in MHCC-97H cells overexpressing miR-30e-5p using qRT-PCR, and found that GRSF1 knockdown did not affect the expression of miR-30e-5p ( $p<0.01$, Fig. 6E). Western bloting assay showed miR-30e-5p inhibited YY1 expression, while GRSF1 knockdown in MHCC-97H cells overexpressing miR-30e-5p further facilitated the downregulation of $Y Y 1$ expression induced by pre-miR-30e-5p ( $p<0.05$, Fig. $6 \mathrm{~F}$ ). RIP assay using an anti-Ago2 antibody demonstrated that overexpressing miR-30e-5p enhanced the interaction between Ago 2 and $Y Y 1$ mRNA, and this interaction was further increased by GRSF1 knockdown ( $p<0.05$, Fig. 6G). Subsequently, qRT-PCR assays demonstrated that anti-miR-30e-5p successfully decreased the expression of miR-30e-5p, while silencing GRSF1 did not lead to the change of miR-30e-5p expression $(p<0.01$, Fig. $6 \mathrm{H})$. Further assays showed that transfection of anti-miR-30e-5p increased YY1 protein expression in MHCC-97H cells, while the silencing GRSF1 disrupted the promotion of YY1 expression caused by anti-miR-30e-5p ( $p<0.05$, Fig. $6 \mathrm{I})$. These results further suggested the competitive effect of GRSF1 and miR-30e-5p on YY1 expression.

In addition, xenograft model in nude mice assay showed that tumors derived from MHCC-97H cells with decreased GRSF1 resulted in smaller tumor sizes (shGRSF1 group) compared with those injected with the control cells (sh-Ctrl group). YY1 overexpression (sh-GRSF1 + ov-YY1group) attenuated the tumor growth $(p<0.05$; Fig. 7A), supporting our results in vitro that GRSF1 promoted HCC upon increased YY1 expression. While xenograft tumors derived from MHCC-97H cells with increased miR-30e-5p resulted in smaller tumor sizes (pre-miR-30e-5p group) compared with those injected with the control cells (pre-NC group). YY1 overexpression (pre-miR-30e-5p + ov-YY1 group) attenuated the tumor growth which had been suppressed by the pre-miR-30e-5p ( $p<0.05$; Fig. $7 C$ ). In addition, xenograft tumors derived from MHCC-97H cells co-transfected with pre-miR-30e-5p and sh-GRSF1 resulted in even smaller tumor sizes (pre-miR-30e-5p + shGRSF1 group) compared with those injected with the cells single transfected with pre-miR-30e-5p (pre-miR-3pe-5p group) ( $p<0.05$; Fig. 7C). And xenograft tumors derived from MHCC-97H cells co-transfected with pre-miR-30e-5p and over-GRSF1 resulted in bigger tumor sizes (pre-miR-30e-5p + ov-GRSF1 group) than those in pre-miR-3pe-5p group ( $p<0.05$; Fig. 7C), supporting our results in vitro that GRSF1 competitively increased YY1 expression in HCC with miR-30e$5 p$.

Immunohistochemistry assay in the HCC xenograft samples showed that GRSF1, YY1 and Ki67 expressions were all decreased in the sh-GRSF1 group tumor tissue, but were markedly recovered in the sh-GRSF1 + ov-YY1 group tumor tissue $(p<0.05$; Fig. $7 \mathrm{~B}$ and E), highlighting that GRSF1 promoted hepatocarcinogenesis upon YY1, and YY1 feedback promoted GRSF1 expression. YY1 and Ki67 expressions were decreased in the pre-miR-30e-5p group tumor tissue compared with the pre-NC group, while were recovered in the pre-miR-30e-5p + ov-YY1 and pre-miR-30e-5p + ov-GRSF1 group tumor tissue ( $p<$ 0.05; Fig. 7D and F).

\section{VE821 inhibits HCC by repressing GRSF1/YY1 pathway}

Among twelve small molecular compounds that may inhibit the growth of HCC cells screened using high-throughput screening technology previously, VE821 (Fig. 8A), a selective inhibitor of the ataxia-telangiectasia-mutated and rad3-related protein (ATR) reduced MHCC-97H and Hep3B proliferation obviously and its function in HCC was rarely reported. Our data showed the half maximal inhibitory concentration (IC50) of VE821 was $38.74 \mu \mathrm{M}$ in MHCC-97H cells and 18.22 $\mu \mathrm{M}$ in Hep3B cells (Fig. 8B). Furthermore, VE821 suppressed GRSF1/YY1 mRNA and protein expression markedly in MHCC-97H and Hep3B cells (Fig. 8C). Hence, we performed assay to evaluate whether VE821 could inhibits HCC by repressing GRSF1/YY1 pathway. The antitumor effect of VE821 on the proliferation of MHCC-97H and Hep3B cells was further verified. As Fig. 8E showed, VE821 inhibited HCC cells proliferation markedly. In addition, we found that VE821 clearly caused cells apoptosis, significantly inhibited cells migration, invasion and colony formation in MHCC-97H cells $(p<0.01$, Fig. 8D). The similar results could be observed in Hep3B cells $(p<0.01$, Fig. 8E).

The xenograft assay in nude mice derived from MHCC-97H cells were randomly divided into two groups and intraperitoneal injection of VE821 or vehicle. The results uncovered that compared with mice treated vehicle, mice treated VE821 exhibited smaller tumor volume and lighter tumor weight ( $p<0.05$, Fig. 8F). Immunohistochemistry assay in the HCC xenograft samples showed GRSF1, YY1 and Ki67 expressions were all decreased in the VE821 group tumor tissue, compared with those in Vehicle group $(p<0.05$; Fig. $8 \mathrm{G})$.In addition, the results of qRT-PCR assay demonstrated that compared with those in Vehicle group, GRSF1, YY1 and Ki67 expressions were decreased in the VE821 group xenograft tumor tissue, while miR-30e-5p expression was increased ( $p<0.01$; Fig. 8H). The results further verified that VE821 prevented HCC progression via inhibiting GRSF1/YY1 signal pathway, providing a novel potential agent for HCC treatment.

To further investigate the underlying mechanism of VE821 in suppressing GRSF1/ YY1 pathway in HCC, we carried out protein degradation analysis in MHCC$97 \mathrm{H}$ cells treated without or with VE821 at different time points. The result demonstrated VE821 obviously impaired the protein stability of GRSF1 ( $p<0.05$, Fin $n \wedge$ and Dl MArmover, RNA stability measurement demonstrated that MHCC-97H cells treated with VE821 exhibited an obviously shorter half-life of YY1 
mRNA compared with those treated without VE821 (NC) $(p<0.05$, Fig. 9C). These data suggested that VE821 inhibited stability of YY1 mRNA via reducing the stability of GRSF1 protein. In addition, VE821 was shown inhibited GRSF1, YY1 expression and promoted miR-30e-5p expression in a dose-dependent manner in MHCC-97H cells $(p<0.01$, Fig. $9 \mathrm{C}$ and D). The similar results could be observed tin Hep3B cells $(p<0.01$, Fig. 9E and F). In short, these results demonstrated that VE821 prevented HCC progression via suppressing GRSF1/YY1 pathway, providing a novel option for HCC treatment.

\section{Discussion}

In this study, we first discovered the oncogenic function of RBP GRSF1 in HCC and the mutual regulating network among GRSF1, YY1 and miR-30e-5p, providing novel mechanistic insight for the pathogenesis of HCC.

The current study elucidated that GRSF1 showed frequently upregulated expression in HCC tissues and cells, was associated with aggressive clinicopathological features of HCC and with a worse clinical outcomes. Similar to our results, GRSF1 is upregulated in cervical cancer and involved in oncogenic activity via regulating miR-G-10 ${ }^{[11]}$ or MIR-G-1 ${ }^{[12]}$. In addition, GRSF1 participates in the miR-346-meditated promotion of cervical cancer cells malignant phenotype via enhancing AGO2 ${ }^{[22]}$. This study revealed that silencing GRSF1 prevented HCC cells malignant biological behaviors in vitro and in vivo, first identifying the protumorigenic factor role of GRSF1 in HCC.

Recently, the elucidation of the interaction networks between RBPs and cancer-related RNA targets has provided a new avenues for the pathogenesis of HCC and attracted growing considerable attention. Latest studies confirmed that a number of RBPs had a significant impact in HCC tumorigenesis via regulating numerous mRNAs at the translational or post-translation level. For example, SORBS2 was shown to inhibit hepatocarcinogenesis by stabilizing RORA expression ${ }^{[7]}$. Zhao et al. revealed RPS3 post-transcriptionally increased its target gene SIRT1 to promote HCC ${ }^{[8]}$. In addition, Chen et al. reported that RDM1 suppressed HCC cells proliferation by targeting p53 ${ }^{[23]}$. Transcriptometric sequencing assay screened a set of GRSF1 target RNAs, and YY1 was identified for further analysis due to its protumorigenic role in HCC. Further results showed that GRSF1 promoted YY1 expression via post-transcriptionally enhancing its mRNA stabilization. Previous researches uncovered that RBPs usually binding to specific sequence region of the target mRNA by its RRM domains. RPS3 is

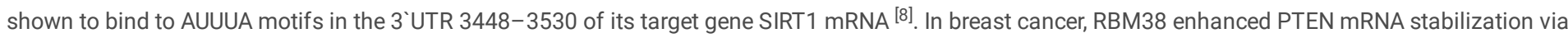
binding to multiple AU/U elements in 3 'UTR of PTEN mRNA ${ }^{[24]}$. In our study, by separating YY1 3UTR into different overlapping fragments, structuring mutants contains different motifs and subsequently performing pull-down assays and luciferase assays, we found that GUUU motifs on YY1 3UTR 26632847 was specific binding region for GRSF1. The rescue assay showed GRSF1 promoted HCC malignant biological behavior depend on oncogene YY1. The results reported here that $Y Y 1$ has importance in promoting hepatocarcinogenesis is consistent with the previous reports. Wu et al. showed $Y Y 1$ transcription factor promoted HCC progression by activating IncMER52A and then stabilized p120-catenin the downstream effector of IncMER52A [25]. YY1 promoted HCC cells proliferation and migration via facilitating linc01134/miR-324-5p/IGF2BP1, and IGF2BP1 stabilizing YY1 mRNA expression, forming a positive feedback loop [26]. Similarly, the facilitating role of $Y Y 1$ in HCC tumorigenesis via eliminating fatty acid oxidation caused by PGC-1 $\beta$ has also been validated ${ }^{\text {[27] }}$. Further data showed YY1 enhanced GRSF1 expression and directly activated GRSF1 promoter. These evidences not only identified that GRSF1 promoted HCC via post-transcriptional regulation of YY1 as an RBP for the first time, but also suggested the interesting role of GRSF1/YY1 positive feedback loop in HCC pathogenesis.

RBPs as well as miRNAs are two common approaches of 3'UTR-dependent gene regulation. Numerous independent studies showed that RBPs could coregulate target gene mRNA with miRNAs. For example, RBP PUM2 is shown to repress osteosarcoma through competitively binding to STARD13 3'UTR against miR-590-3p and miR-9 ${ }^{[28]}$. Similarly, transformer $2 \beta$ could bind to BCL2 $3^{\prime} U T R$ mRNA and increase its stabilization via antagonizing the binding of miR-548-3p ${ }^{[29]}$. The RBP HuR promotes stemness of lung cancer cells by functionally opposing the miR-873 and miR-125a-3p, thus competitively binds to CDK3 mRNA ${ }^{[30]}$. Similar finding in another study showed HuR and miR-494 functionally competed for binding with nucleolin $3^{\prime} U T R$ in cervical carcinoma, and thus HuR facilitates cervical carcinoma growth [31]. These results verified that RBPs could mediate target mRNAs via the joint regulation of miRNAs, when binding regions for miRNAs overlap with those for RBPs. In our study, bioinformatic analysis results showed binding sites for miR-30e-5p on YY1 mRNA are partially overlap with GRSF1-binding sites. Further assays confirmed that GRSF1 used one GUUU sequence within the binding sites onYY1 3'UTR for miR-30e$5 p$, and antagonize miR-30e-5p to regulate $Y Y 1$ expression. We thus discovered a novel crosstalk between GRSF1 and miR-30e-5p during HCC tumorigenesis, providing further understand of network of hepatocarcinogenesis. We have to mention that the regulatory mechanism for the abnormal expression of YY1 in HCC is very likely be more complicated than only co-regulation between GRSF1 and miR-30e-5p. However, the competitive regulation between GRSF1 and miR$30 \mathrm{e}-5 \mathrm{p}$ provide novel insight into the regulatory mechanism for YY1 in HCC. Besides, the tumor suppressor role of miR-304-5p in HCC discovered in this current study is consistent with the reports of previous studies. Hu et al. reported that miR-30e-5p is frequently downregulated in nasopharyngeal carcinoma (NPC), miR-30e-5p targets oncogene MTA1, and thus inhibited NPC cells migration ${ }^{[32]}$. miR-30e-5p also could target AEG-1, which was implicated in the angiogenesis and metastasis of Squamous Cell Carcinoma of the Head and Neck (SCCHN), suggesting that miR-30e-5p is a novel biomarker and target in SCCHN [33]. Downregulated miR-30e-5p leads to increase its target gene oncogene MTDH, and thereby promotes bladder cancer cells growth and invasiveness ${ }^{\text {[3] }}$.

Though HCC systemic treatment options have been expanded, including vascular endothelial growth factor receptor, tyrosine kinase inhibitors and immune check-point inhibitors, the clinical prognosis remains disturbingly poor ${ }^{[35,36]}$. Immunotherapy is a promising regimens that has been used in clinical treatment [37]. However, their efficacy are limited in a part of patients. Even more confusing, it is not easy to identify the patients who may benefit from immunotherapy. Some serious side effects and the high financial cost also limited their application ${ }^{[38]}$. Hence, further researches directed toward developing additional active, and safe approaches regard to HCC remain urgent. In this study, we identified VE-821 inhibited HCC growth in vitro and in vivo. Our results is in accordance with antitumor agent role of VE821 in other researches. Dias et al reported VE-821 could induced cancer cells death ${ }^{[39]}$. Another report also uncovered VE-821 induced BRCA1 Mutant Ovarian Cells death ${ }^{[40]}$. The data in this study uncovered that VE-821 prevented HCC progression by repressing GRSF1/YY1 axis and pabnonad min $0 n_{n}$ Ep expression. However, whether VE-821 could be used as a novel clinical treatment option for HCC patients deservedly requires deeper 
researches. In addition, recent research suggested that VE-821 showed a synergistic effect with PD-L1 inhibition in pancreatic cancer, and PD-L1 inhibition increased VE-821 sensitivity ${ }^{[40]}$. GRSF1 is necessary in the progression of ATR-dependent regulation on Dux, which is vital for the cleavage-specific transcription ${ }^{[41]}$. Therefore, whether the combination with PD-L1 inhibition could improve antitumor function of VE-821 on HCC induced by GRSF1 is worth to be further discussed in the future.

\section{Conclusions}

Taken together, this study first measure the expression pattern and function of GRSF1, YY1, miR-30e-5p in HCC and investigated the mutual regulating network among them. We revealed GRSF1 worked as a novel oncogenic RBP in HCC by stabilizing oncogene YY1 mRNA. YY1 feedback promoted GRSF1 expression by binding to GRSF1 promoters. GRSF1 and miR-30e-5p competitively regulated YY1 via binding to its YY1 3'UTR 2663-2847 region. Furthermore, we discovered that 3-amino-6-(4-methylsulfonylphenyl)-N-phenylpyrazine-2-carboxamide, VE-821, could block HCC tumorigenesis by inhibiting GRSF1/YY1 axis. Our data provide novel mechanistic insight for the pathogenesis of HCC, and identified an additional promising therapeutic target of HCC.

\section{Abbreviations}

RBPS

RNA Binding Protein

GRSF1

G-rich sequence binding factor 1

YY1

Yin-Yang 1

$\mathrm{HCC}$

Hepatocellular Carcinoma

OS

Overall Survival

DFS

Disease free survival

VE821

3-amino-6-(4-methylsulfonylphenyl)-N-phenylpyrazine-2-carboxamide

\section{Declarations}

\section{Ethics approval and consent to participate}

All applicable international, national, and/or institutional guidelines for the care and use of animals were followed.All procedures performed in studies involving human participants were in accordance with the ethical standards of the institutional and/or national research committee and with the 1964 Helsinki declaration and its later amendments or comparable ethical standards.

\section{Consent for publication}

All authors agreed on the manuscript.

\section{Availability of data and material}

The datasets supporting the conclusions of this article are included within the article and its Additional files.

\section{Competing interests}

All authors have no conflicts of interest.

\section{Funding}

This study was funded by the Natural Science Foundation of Shaanxi Province (No.2019JQ-128), the China Postdoctoral Science General Financial Grant (No. 2017M623193).

\section{Authors' contributions}

Conception and design: Lili Han

Acquisition of data: LiliHan ,Shuqun Zhang

Analysis and interpretation of dat: Xiaofei Wang

Writing, review and revision of the manuscript:Lili Han,Fei Wu, Lumin Wang

Processing math: $14 \%$ haterial support: Chen Huang, Shuqun Zhang

Page $10 / 21$ 


\section{Acknowledgements}

We are grateful for participation and cooperation from HCC patients.

\section{References}

1. Finn RS. Current and future treatment strategies for patients with advanced hepatocellular carcinoma: role of mTOR inhibition. Liver Cancer. 2012;3:24756.

2. Kew MC. Epidemiology of chronic hepatitis B virus infection, hepatocellular carcinoma, and hepatitis B virus-induced hepatocellular carcinoma. PatholBiol. 2010;58:273-7.

3. Kudo M. Systemic Therapy for Hepatocellular Carcinoma: 2017 Update. Oncology. 2017; 93.

4. Gerstberger S, Hafner M, Tuschl T. A census of human RNA-binding proteins. Nat Rev Genet. 2014;15:829-45.

5. Glisovic T, Bachorik JL, Yong J, Dreyfuss G. RNA-binding proteins and post-transcriptional gene regulation. FEBS Lett. 2008;582:1977-86.

6. Dreyfuss G, Kim VN, Kataoka N. Messenger RNA binding proteins and the messages they carry. Nat Rev Mol Cell Biol. 2002;3:195-205.

7. Han L, Huang C, Zhang S. The RNA-binding protein SORBS2 suppresses hepatocellular carcinoma tumourigenesis and metastasis by stabilizing RORA mRNA. Liver Int. 2019;39(11):2190-203.

8. Zhao L, et al. RNA-binding protein RPS3 contributes to hepatocarcinogenesis by post-transcriptionally up-regulating SIRT1. Nucleic Acids Res. 2019;47:2011-28.

9. Galante PA, et al. A comprehensive in silico expression analysis of RNA binding proteins in normal and tumor tissue: Identification of potential players in tumor formation. RNA Biol. 2009;6:426-33.

10. Noh JH, Kim KM, Pandey PR, et al. Loss of RNA-binding protein GRSF1 activates mTOR to elicit a proinflammatory transcriptional program. Nucleic Acids Res. 2019;47(5):2472-86.

11. Sun Q, Yang Z, Li P, et al. A novel miRNA identified in GRSF1 complex drives the metastasis via the PIK3R3/AKT/NF-KB and TIMP3/MMP9 pathways in cervical cancer cells. Cell Death Dis. 2019;10(9):636.

12. Yang Z, Sun Q, Guo J, et al. GRSF1-mediated MIR-G-1 promotes malignant behavior and nuclear autophagy by directly upregulating TMED5 and LMNB1 in cervical cancer cells. Autophagy. 2019;15(4):668-85.

13. Wang J, Zhou L, Li Z, Zhang T, Liu W, Liu Z, et al. YY1 suppresses FEN1 over-expression and drug resistance in breast cancer. BMC Cancer. $2015 ; 15: 50$.

14. Bonavida B, Kaufhold S. Prognostic significance of YY1 protein expression and mRNA levels by bioinformatics analysis in human cancers: a therapeutic target. Pharmacol Therapeut. 2015;150:149-68.

15. Shi JM, Hao AX, Zhang Q, Sui GC. The role of YY1 in oncogenesis and its potential as a drug target in cancer therapies. Curr Cancer Drug Tar. 2015;15:145-57.

16. Jin F, Wang Y, Li M, et al. MiR-26 enhances chemosensitivity and promotes apoptosis of hepatocellular carcinoma cells through inhibiting autophagy. Cell Death Dis. 2017;8(1):e2540.

17. Gu H, Gu S, Zhang X, et al. miR-106b-5p promotes aggressive progression of hepatocellular carcinoma via targeting RUNX3. Cancer Med. 2019;8(15):6756-67.

18. Han LL, Yin XR, Zhang SQ. miR-103 promotes the metastasis and EMT of hepatocellular carcinoma by directly inhibiting LATS2. Int J Oncol. 2018;53(6):2433-44.

19. Hu W, Yao W, Li H, Chen L. MiR-30e-5p inhibits the migration and invasion of nasopharyngeal carcinoma via regulating the expression of MTA1. Biosci Rep. 2020;40(5):BSR20194309.

20. Cai J, Wang L, et al. MicroRNA-30e-5p suppresses non-small cell lung cancer tumorigenesis by regulating USP22-mediated Sirt1/JAK/STAT3 signaling. Exp Cell Res. 2018;362(2):268-78.

21. Saleh AD, Cheng H, Martin SE, et al. Integrated Genomic and Functional microRNA Analysis Identifies miR-30-5p as a Tumor Suppressor and Potential Therapeutic Nanomedicine in Head and Neck Cancer. Clin Cancer Res. 2019;25(9):2860-73.

22. Guo J, Lv J, Liu M, Tang H. miR-346 Up-regulates Argonaute 2 (AGO2) Protein Expression to Augment the Activity of Other MicroRNAs (miRNAs) and Contributes to Cervical Cancer Cell Malignancy. J Biol Chem. 2015;290(51):30342-50.

23. Chen SL, Liu LL, Wang CH, et al. Loss of RDM1 enhances hepatocellular carcinoma progression via p53 and Ras/Raf/ERK pathways. Mol Oncol. 2020;14(2):373-86.

24. Zhou XJ, Wu J, Shi L, et al. PTEN expression is upregulated by a RNA-binding protein RBM38 via enhancing its mRNA stability in breast cancer. J Exp Clin Cancer Res. 2017;36(1):149.

25. Wu Y, Zhao Y, Huan L, et al. An LTR Retrotransposon-Derived Long Noncoding RNA IncMER52A Promotes Hepatocellular Carcinoma Progression by Binding p120-Catenin. Cancer Res. 2020;80(5):976-87.

26. Rong Z, Wang Z, Wang X, Qin C, Geng W. Molecular interplay between linc01134 and YY1 dictates hepatocellular carcinoma progression. J Exp Clin Cancer Res. 2020;39(1):61.

27. Li Y, Kasim V, Yan X, et al. Yin Yang 1 facilitates hepatocellular carcinoma cell lipid metabolism and tumor progression by inhibiting PGC-1 3 -induced fatty acid oxidation. Theranostics. 2019;9(25):7599-615. 
28. Hu R, Zhu X, Chen C, Xu R, Li Y, Xu W. RNA-binding protein PUM2 suppresses osteosarcoma progression via partly and competitively binding to STARD13 3'UTR with miRNAs. Cell Prolif. 2018;51(6):e12508.

29. Kuwano Y, Nishida K, Kajita K, et al. Transformer $2 \beta$ and miR-204 regulate apoptosis through competitive binding to 3 ' UTR of BCL2 mRNA. Cell Death Differ. 2015;22(5):815-25.

30. Zhang Y, Yang L, Ling C, Heng W. HuR facilitates cancer stemness of lung cancer cells via regulating miR-873/CDK3 and miR-125a-3p/CDK3 axis. Biotechnol Lett. 2018;40(4):623-31.

31. Tominaga K, Srikantan S, Lee EK, et al. Competitive regulation of nucleolin expression by HuR and miR-494. Mol Cell Biol. 2011;31(20):4219-31.

32. Hu W, Yao W, Li H, Chen L. MiR-30e-5p inhibits the migration and invasion of nasopharyngeal carcinoma via regulating the expression of MTA1. Biosci Rep. 2020;40(5):BSR20194309.

33. Zhang S, Li G, Liu C, et al. miR-30e-5p represses angiogenesis and metastasis by directly targeting AEG-1 in squamous cell carcinoma of the head and neck. Cancer Sci. 2020;111(2):356-68.

34. Zhang Z, Qin H, Jiang B, et al. miR-30e-5p suppresses cell proliferation and migration in bladder cancer through regulating metadherin. J Cell Biochem. 2019;120(9):15924-32.

35. Marrero JA, Kulik LM, Sirlin CB, et al. Diagnosis, Staging, and Management of Hepatocellular Carcinoma: 2018 Practice Guidance by the American Association for the Study of Liver Diseases. Hepatology. 2018;68(2):723-50.

36. Lee HW, Cho KJ, Park JY. Current Status and Future Direction of Immunotherapy in Hepatocellular Carcinoma: What Do the Data Suggest? Immune Netw. 2020;20(1):e11.

37. Schreiber RD, Old LJ, Smyth MJ. Cancer immunoediting: integrating immunity's roles in cancer suppression and promotion. Science. 2011;331(6024):1565-70.

38. Iñarrairaegui M, Melero I, Sangro B. Immunotherapy of Hepatocellular Carcinoma: Facts and Hopes. Clin Cancer Res. 2018;24(7):1518-24.

39. Dias MH, Fonseca CS, Zeidler JD, et al. Fibroblast Growth Factor 2 lethally sensitizes cancer cells to stress-targeted therapeutic inhibitors. Mol Oncol. 2019;13(2):290-306.

40. Burgess BT, Anderson AM, McCorkle JR, Wu J, Ueland FR, Kolesar JM. Olaparib Combined with an ATR or Chk1 Inhibitor as a Treatment Strategy for Acquired Olaparib-Resistant BRCA1 Mutant Ovarian Cells. Diagnostics (Basel). 2020;10(2):121.

41. Atashpaz S, Samadi Shams S, Gonzalez JM, et al. ATR expands embryonic stem cell fate potential in response to replication stress. Elife. 2020;9:e54756.

\section{Figures}


A
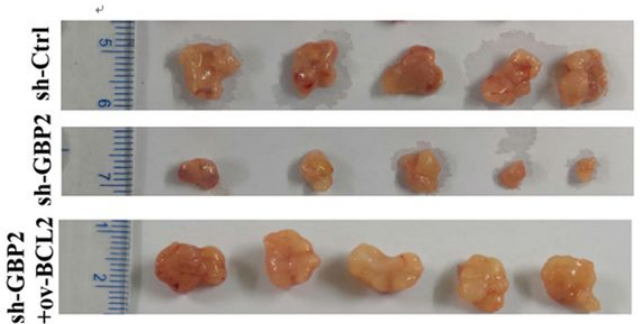

B

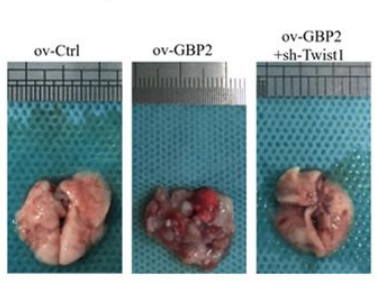

C
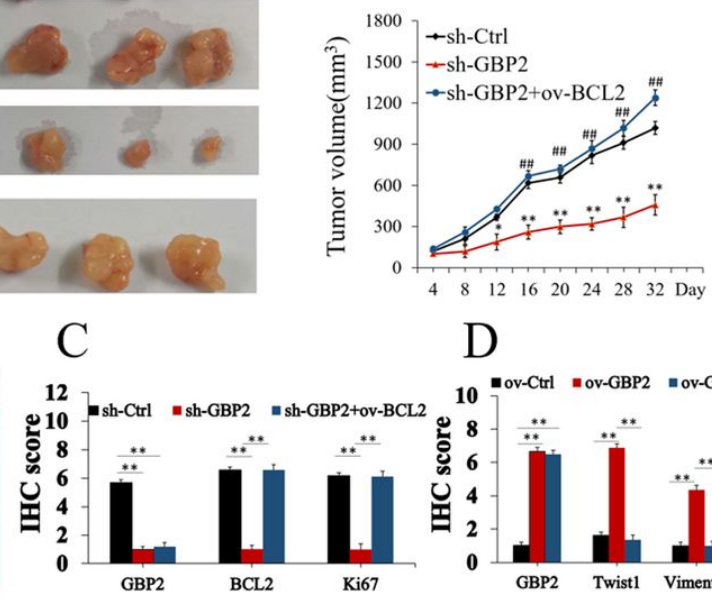

D
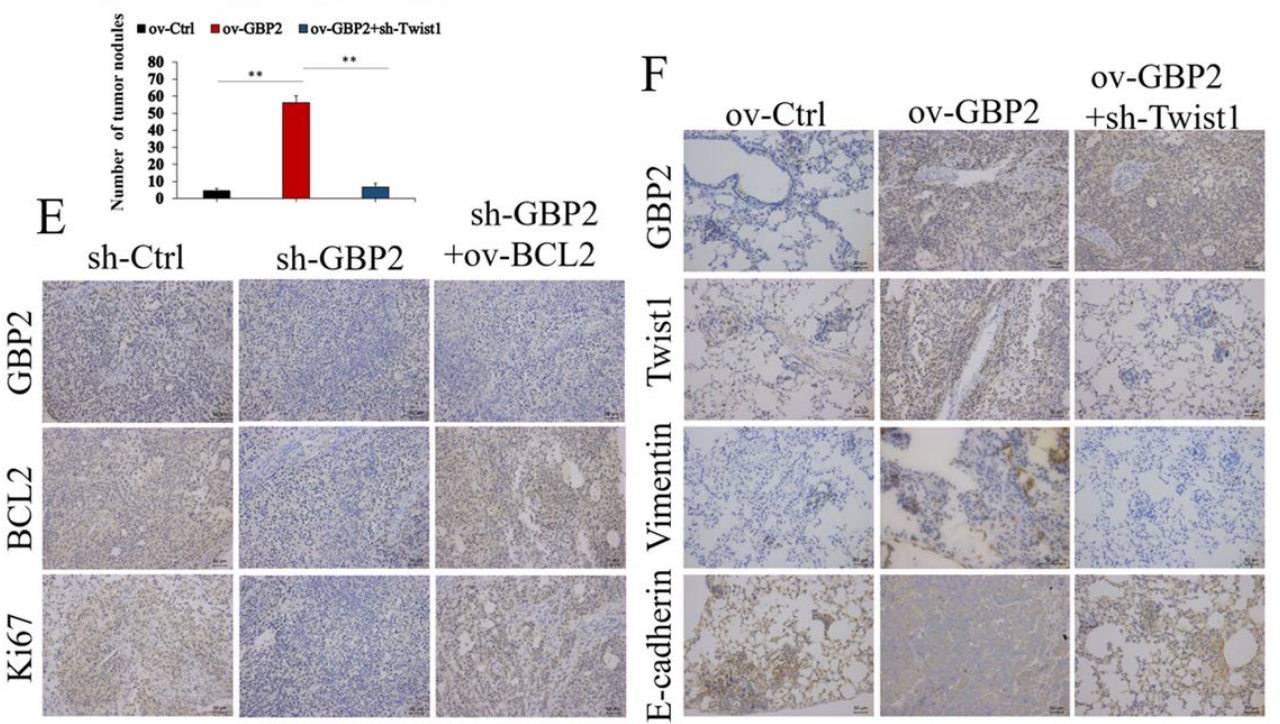

\section{Figure 1}

VE821 inhibits HCC by repressing GRSF1/YY1 pathway. A. B. CHX chase assay showed GRSF1 expression in MHCC-97H cells treated with VE821 ornegative control. C.VE821 shorten YY1 mRNA half-life. D. GRSF1, YY1 and miR-30e-5p mRNA expression in MHCC-97H cells treated with different concentrations of VE821.E. GRSF1 and YY1 and protein expression in MHCC-97H cells treated with different concentrations of VE821. F. GRSF1, YY1 and miR-30e-5p mRNA expression in Hep3B cells treated with different concentrations of VE821.G. GRSF1and YY1 and protein expression in Hep3B cells treated with different concentrations of VE821. ${ }^{\star} \mathrm{p}<0.05 \llbracket \star * \mathrm{p}<0.01$. 


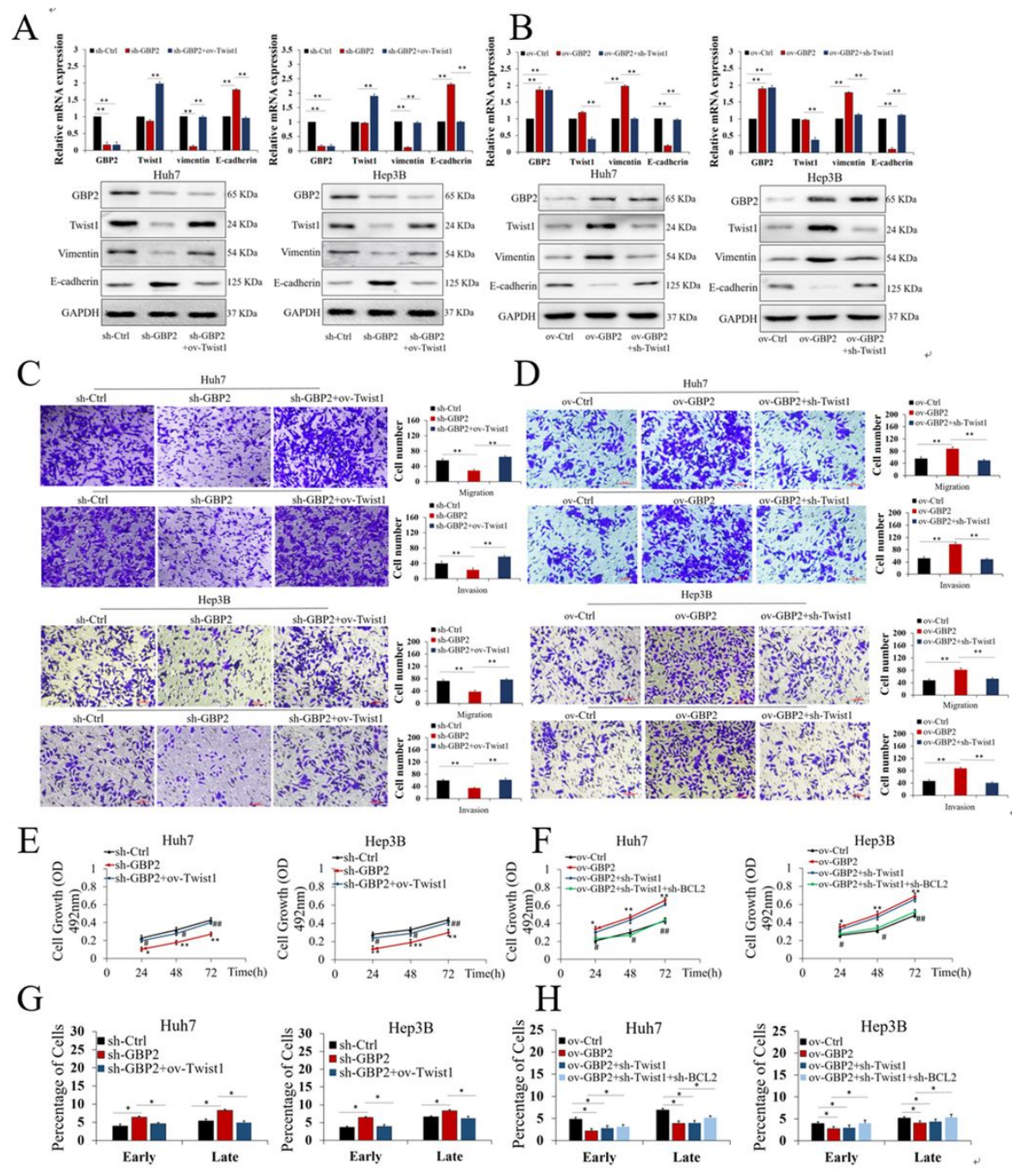

Figure 2

VE821 inhibits HCC by repressing GRSF1/YY1 pathway. A. Chemical structure of VE821. B. Cytotoxicity analysis of VE821 in MHCC-97H and Hep3B cells. C. GRSF1 and YY1 expression in MHCC-97H and Hep3B cells treated with VE821 or negative control. D.VE821 markedly inhibited cells proliferation, migration, invasion and colony formation ability, and enhanced cells apoptosis of MHCC-97H cells. E. VE821 markedly inhibited malignant biological behavior of Hep3B cells. F. Representative images of the resected subcutaneous tumors from VE821 or Vehicle group. G. IHC assay showed GRSF1, YY1 and Ki67 expression in the xenografttumor tissue in VE821 or Vehicle group (magnification, 200x). H. GRSF1, YY1, Ki67 and miR-30e-5p mRNA expression in the xenografttumor tissue in VE821 or Vehicle group. ${ }^{*} p<0.05{ }^{\star *} \mathrm{p}<0.01$. 

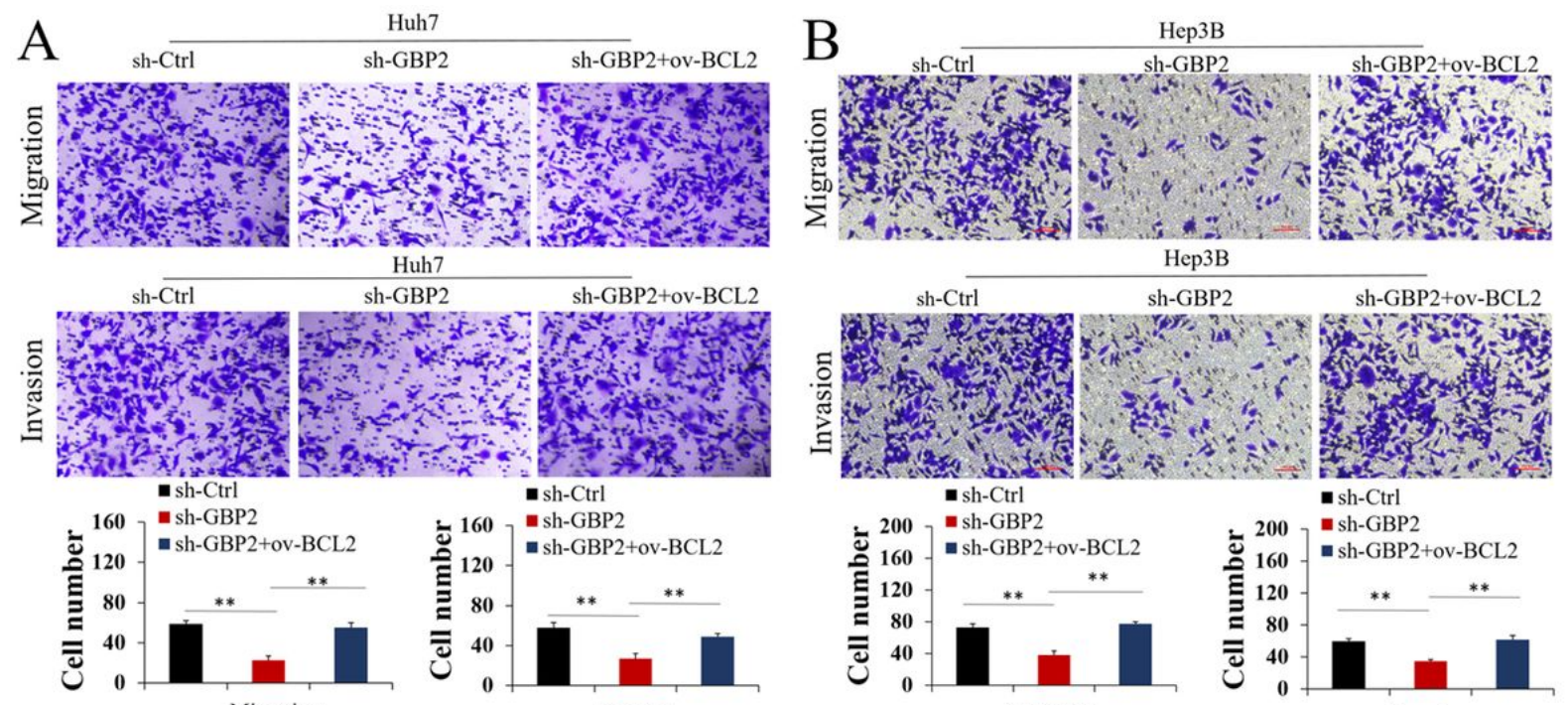

Huh7

sh-GBP2+ov-BCL2
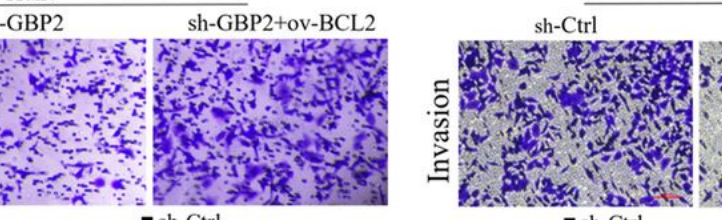

sh-GBP2

sh-GBP2+ov-BCL2

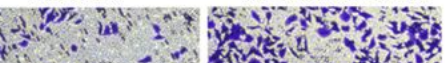

C
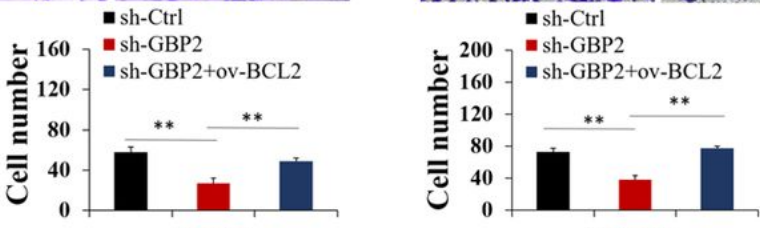

D
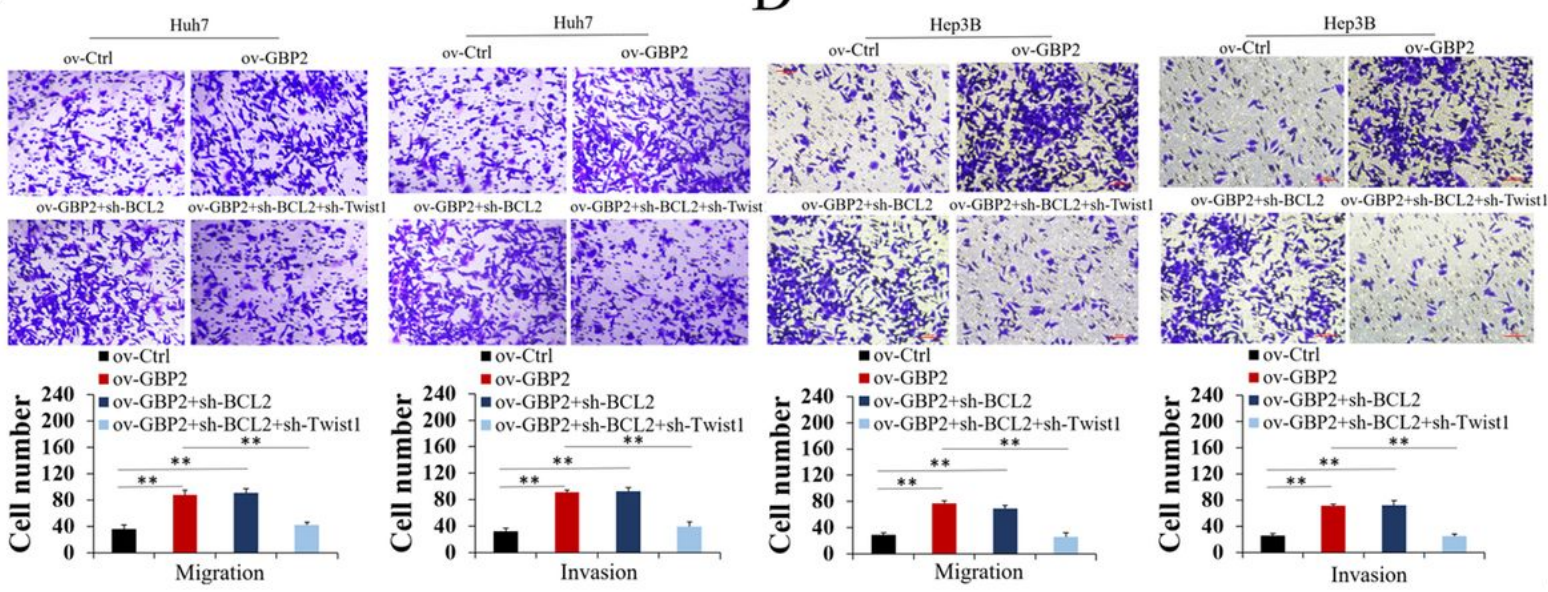

Figure 3

The regulatory network of GRSF1, miR30e-5p and YY1 was explored in vivo.A. Decreased GRSF1 suppressed HCC xenografttumor growth ( ${ }^{\star} p<0.05$, ${ }^{*} p<0.01$ ), while YY1 overexpression counteracted the inhibitory effect of sh-GRSF1 (\#p<0.05, \#\#p<0.01). B.E. IHC assay showed GRSF1, YY1 and Ki67 expression in the xenografttumor tissue in different groups (magnification, 200x, ${ }^{\mathrm{p}}<0.05$ ). C.Overexpressed miR-30e-5p suppressed HCC xenografttumor growth ( $<<0.05$ ), YY1 overexpression or GRSF1 overexpression attenuated the inhibitory effect induced by pre-miR-30e-5p ( $<<0.05)$, co-transfection with pre-miR-30e-5p and shGRSF1 resulted in even smaller tumor sizes than those transfected with pre- miR-30e-5p. D.F. IHC assay showed GRSF1, YY1 and Ki67 expression in the xenografttumor tissue in different groups (magnification, 200x, ${ }^{*} p<0.05$ ). 

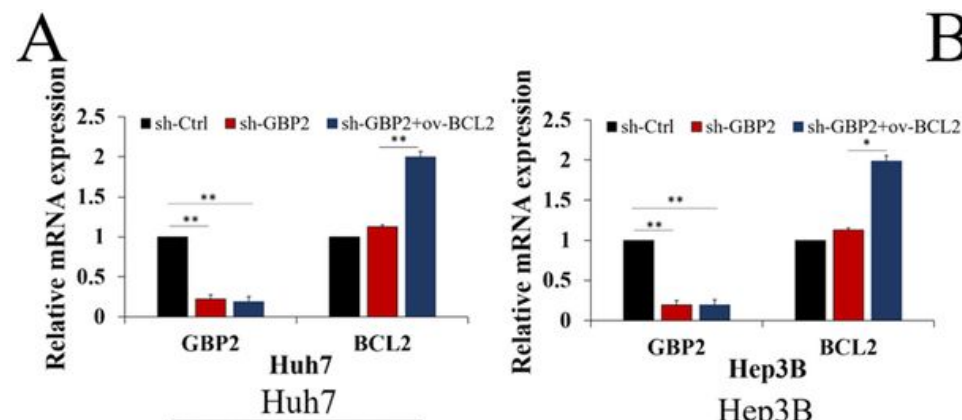

$\mathrm{B}$
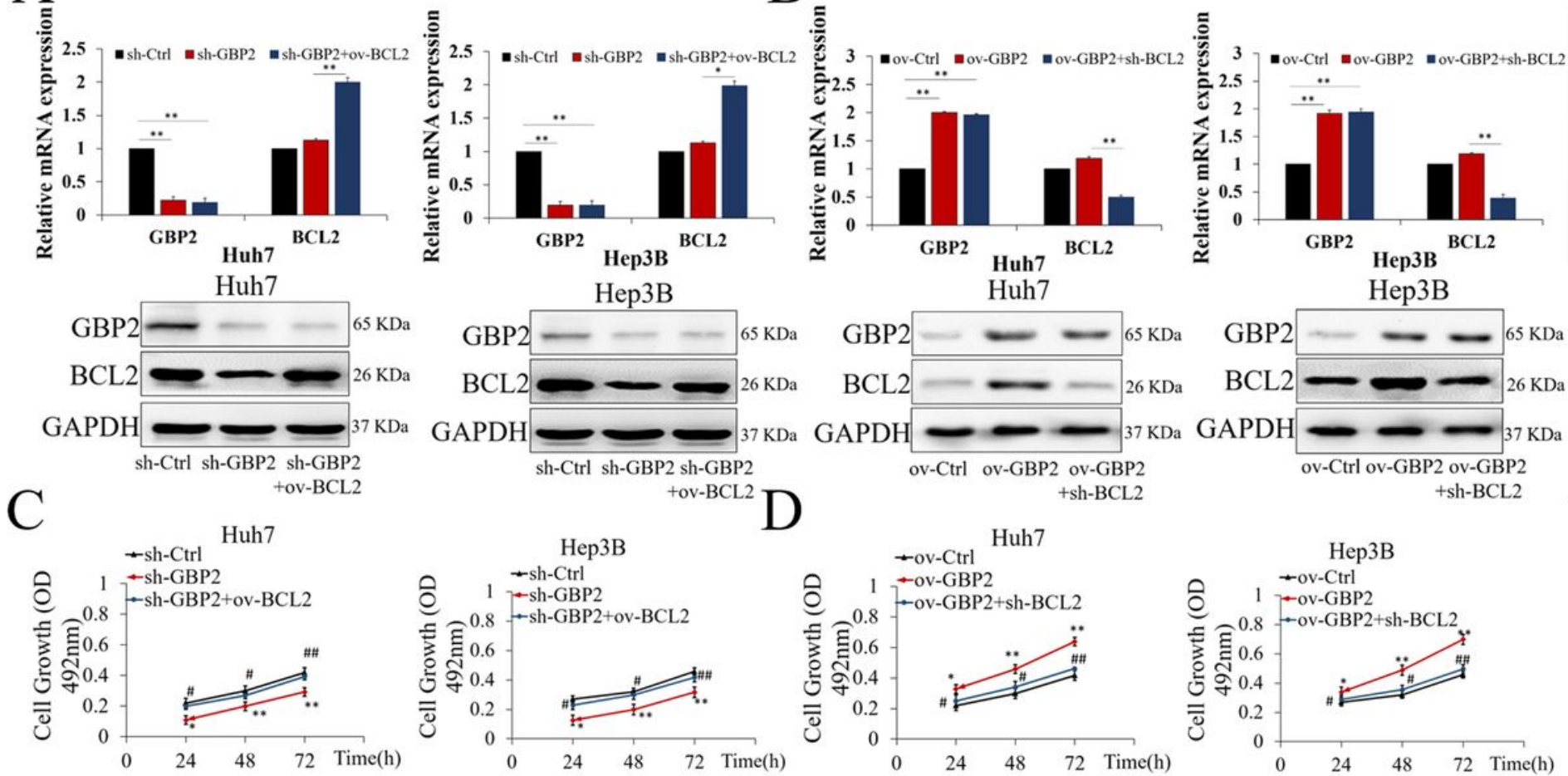

$\mathrm{E}$
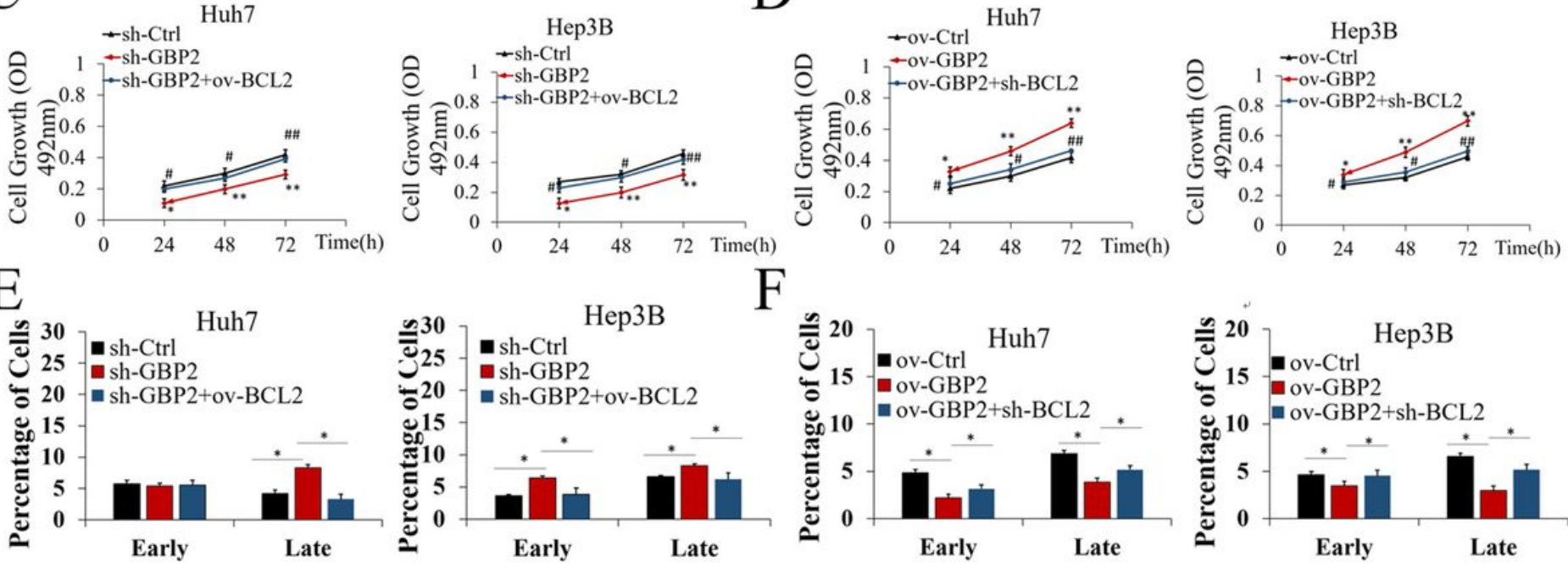

$\mathrm{F}$
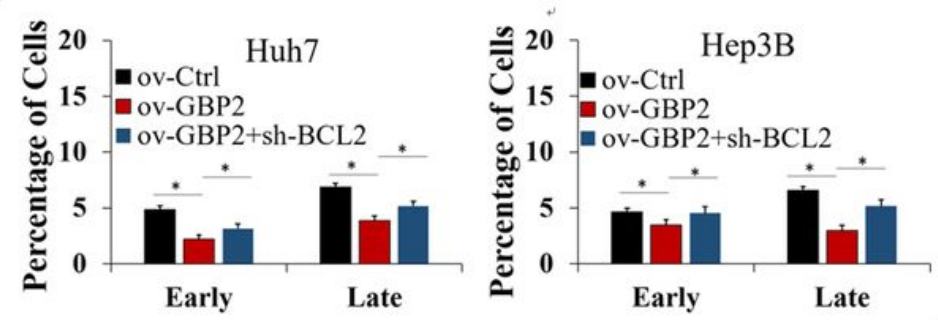

Figure 4

GRSF1 and miR-30e-5p competitively regulated YY1 via binding to its 3UTR. A.B. Wild-type fragment (WT) of YY1 3UTR 2663-2847and four mutants were structured and used inRNA pull-down assays. C. YFP reporter construct only containing with YFP (YFP Ctrl), wild-type YY13 'UTR (YFP-YY1 WT), mutatedmiR30e-5p-binding site (YFP-YY1-miR-30e-5p MT), or mutated GRSF1-binding site (YFP-YY1-GRSF1 MT). The underlined sequences were the mutated sites. D. YFP Ctrl, YFP-YY1 WT, YFP-YY1-miR-30e-5p MT or YFP-YY1-GRSF1 MTconstruct was transfected into MHCC-97H cells and then YFP mRNA enrichment in Ago2-IP was analyzed by RIP assay followed by qPCR. E.miR-30e-5p expression in MHCC-97H cells co-transfected with pre-miR-30e-5p and sh-GRSF1 vector. F.Western blot assay showed pre-miR-30e-5p decreased YY1 expression, knockdown of GRSF1 further enhance this downregulation of YY1 expression. G. RIP assay showed pre-miR-30e-5p enhanced the enrichment of YY1 mRNA in Ago2-IP, which was further enhanced by GRSF1 knockdown.H. miR-30e-5p expression in MHCC-97H cells co-transfected with anti-miR-30e-5p and sh-GRSF1 vector.l. GRSF1 and YY1 expression in MHCC-97H cells co-transfected with pre-miR-30e-5p and sh-GRSF1 vector. ${ }^{*} \mathrm{p}<0.05 \mathbb{\Xi}^{\star *} \mathrm{p}<0.01$. 

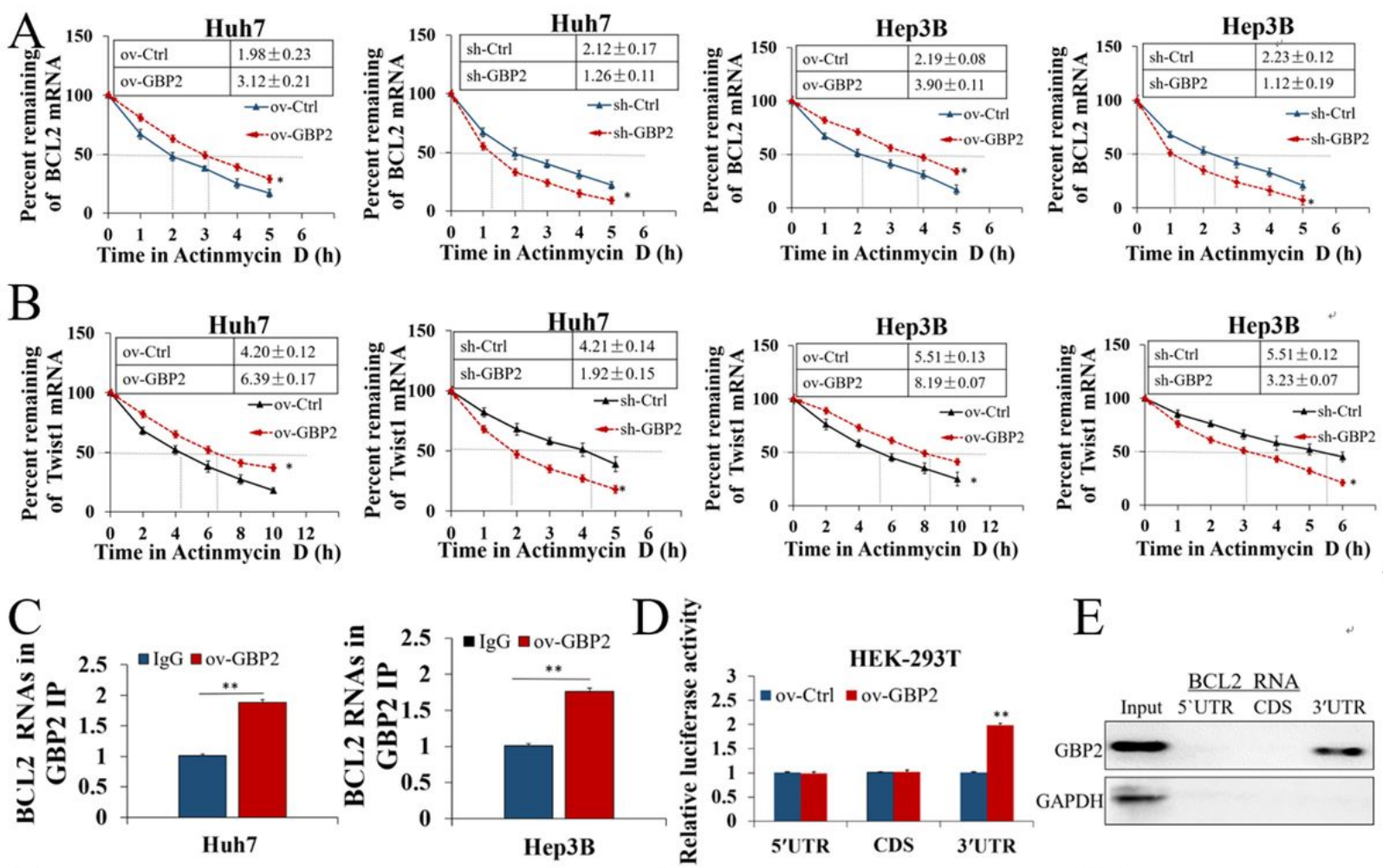

E
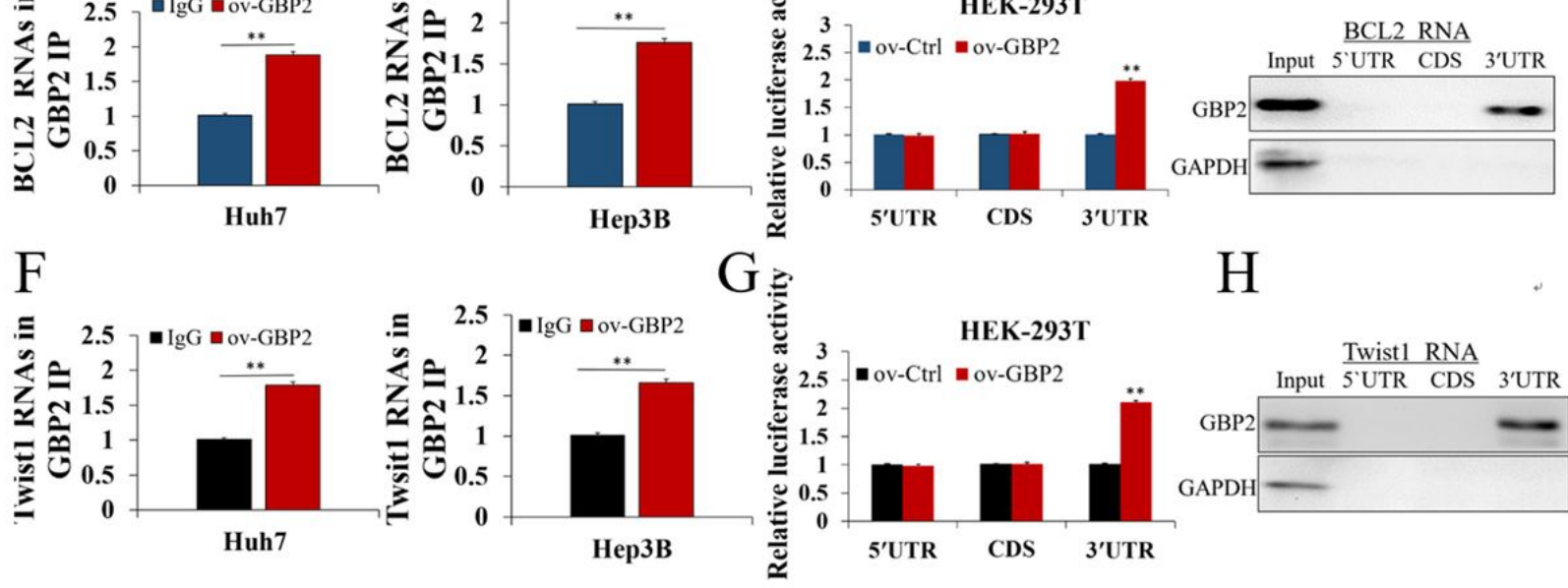

$\mathrm{H}$

\section{Figure 5}

miR-30e-5p inhibited $Y Y 1$ and hepatocarcinogenesis via binding with 3'UTR of YY1. A. miR-30e-5p and its putative binding sequence in YY1 3'-UTR. B. miR$30 \mathrm{e}-5 \mathrm{p}$ expression in $120 \mathrm{HCC}$ and paired non-cancerous tissues. C.miR-30e-5p expression in MHCC-97H and Hep3B cells was increased upon the transfection with pre-miR-30e-5p, but was not regulated by $Y Y 1$ overexpression. D.Luciferase reporter gene assay showed the miR-30e-5p overexpression decreased $Y Y 1$ luciferase activity carried with a wt YY1 3'-UTR.E. qRT-PCR assay and western blot assays showed YY1 expression was decreased followed with increased miR-30e-5p and upregulated upon transfection with ov-YY1 vector in MHCC-97H and Hep3B cells. F-H. Increased miR-30e-5psuppressed the ability of colonforming, migration, invasion and proliferation ofMHCC-97H and Hep3B cells, and the anti-tumor function of miR-30e-5p was counteracted by YY1overexpression. ${ }^{\star} \mathrm{p}<0.05 \rrbracket^{\star *} \mathrm{p}<0.01$. 


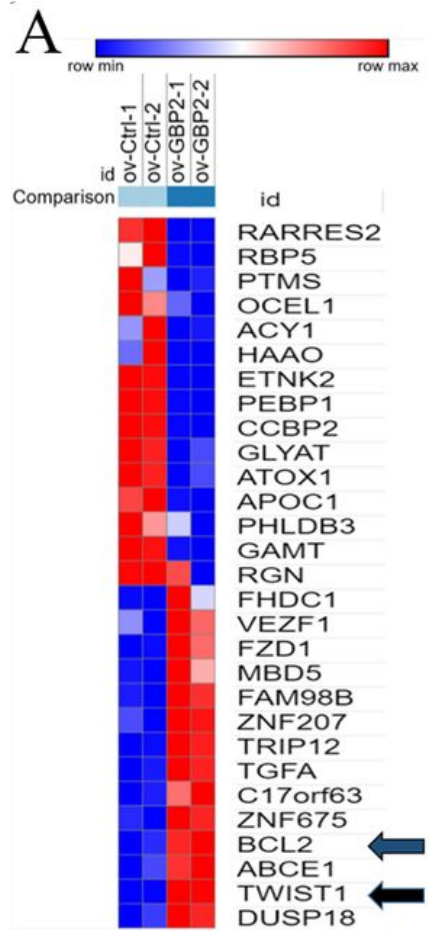

$\mathrm{D}$
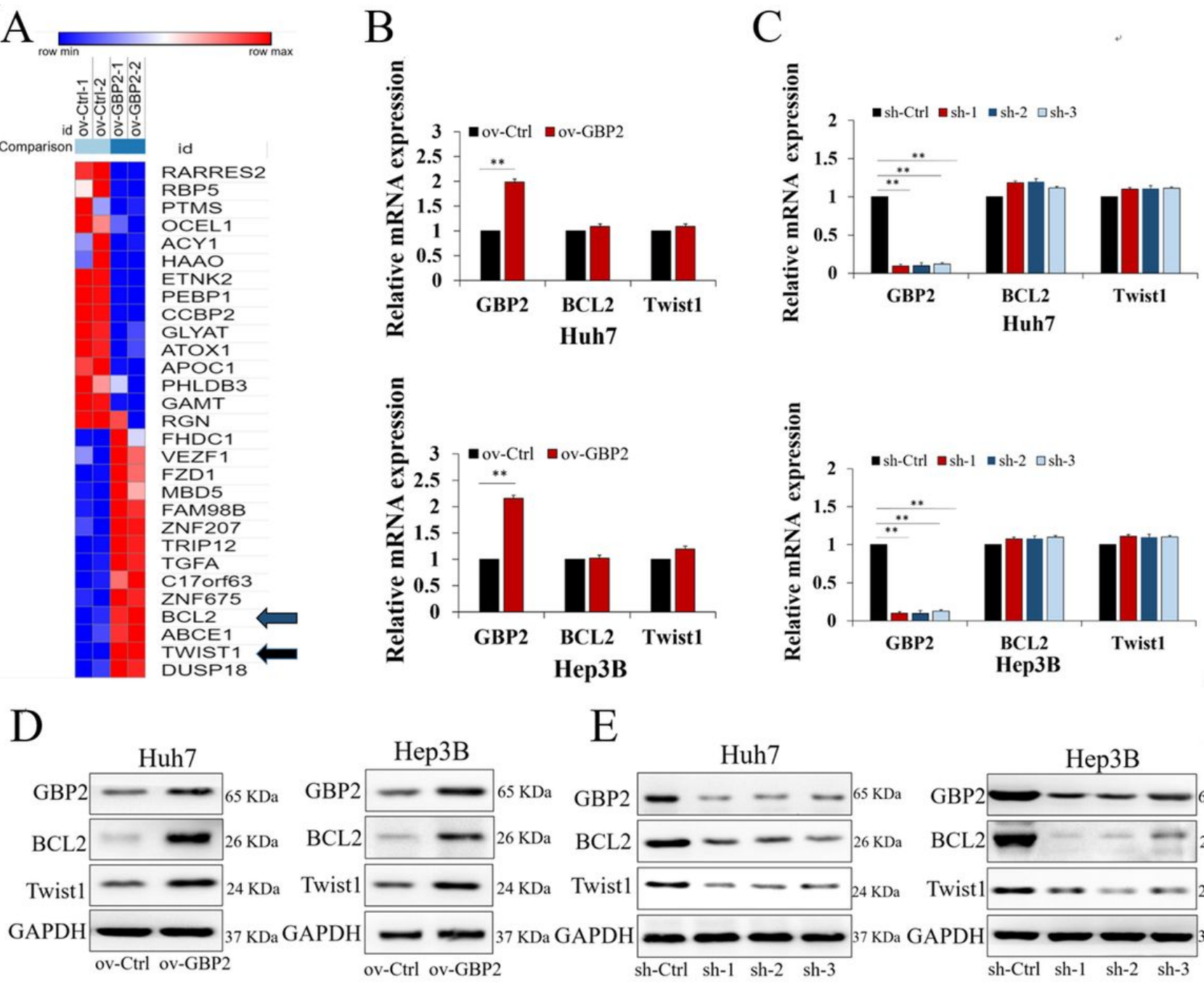

\section{Figure 6}

YY1 worked as an essential target of GRSF1 and feedback promoted GRSF1 expression.A. GRSF1 and YY1 expression in MHCC-97H and Hep3B cells cotransfected with sh-GRSF1 and ov-YY1 vectors. B-E.YY1 overexpression rescued tumor-inhibiting effect caused by GRSF1 knockdown on cells proliferation, colony formation ability, cell apoptosis, invasion and migration ability of MHCC-97H and Hep3B cells. The invaded cells in Transwell assays were quantified by counting the cells in 10 random fields (magnification, 200x). F. GRSF1 mRNA expression levels were decreased followed YY1 knockdown, while was increased after YY1 overexpression in MHCC-97H and Hep3B. G. GRSF1 protein expression levels also changed with the expression of YY1 in MHCC-97H and Hep3B.H.ChIP assays showed that $Y Y 1$ bound to the promoter of GRSF1, but not to the control region. ${ }^{*} p<0.05 \rrbracket^{\star *} p<0.01$. 
A

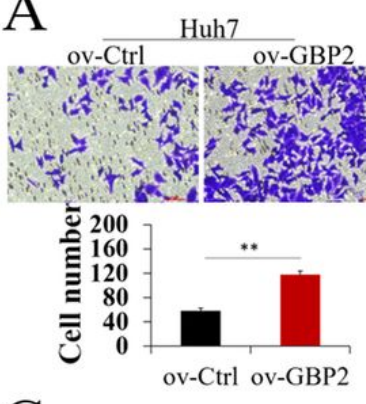

$\mathrm{C}$
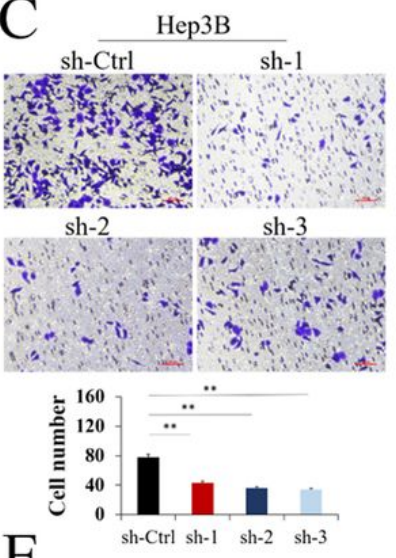

E

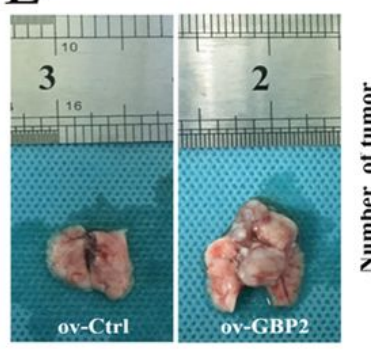

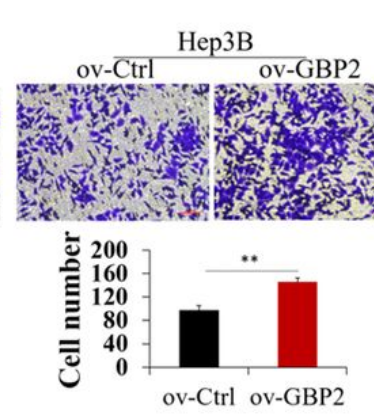
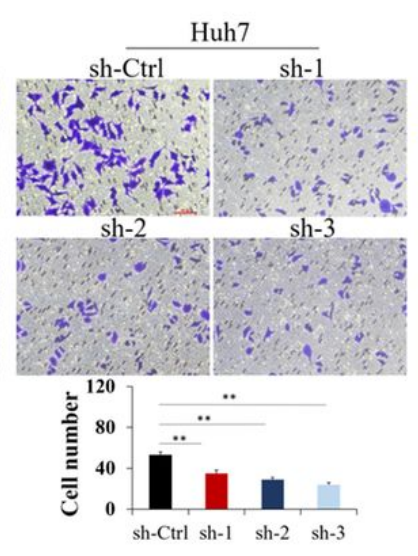

B Huh7

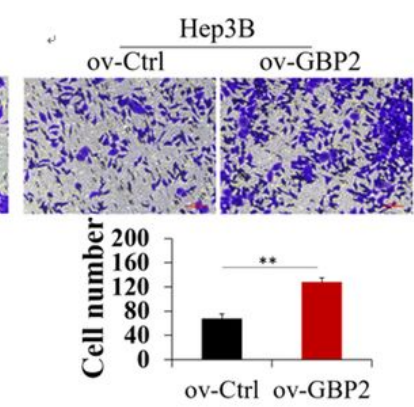

D

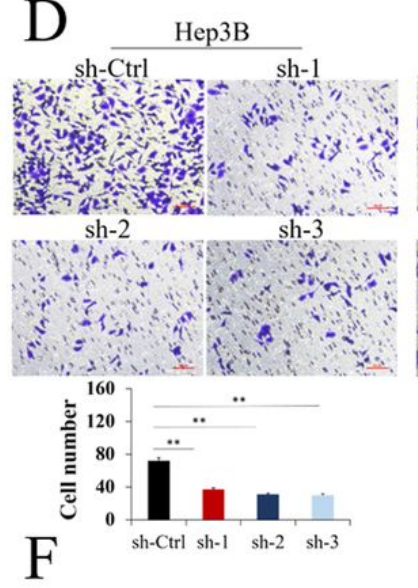

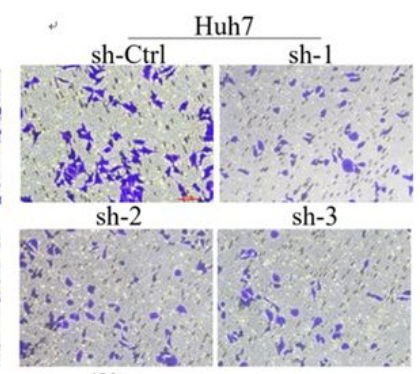

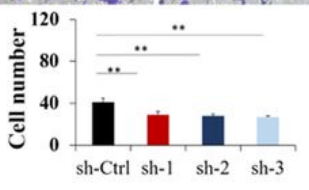

\section{Figure 7}

GRSF1 enhanced YY1 expression via interacting with YY1 mRNA. A. Heat map representing genes with dramatic log2-transformed fold changes induced by GRSF1 knockdown in MHCC-97H cells. B. YY1 mRNA expression levels in 120 HCC and paired non-cancerous tissues. C.GRSF1and YY1 mRNA expression in GSRF1-deficient MHCC-97H and Hep3B cells. D. GRSF1and YY1 protein expression in GSRF1- deficient MHCC-97H and Hep3B cells. E. RIP assays showed the direct interaction between GRSF1 and YY1. F. Silencing GRSF1 markedly shortened the half-life of YY1 mRNA in MHCC-97H and Hep3B cells. G-J. The specific binding region on YY1 mRNA for GRSF1 was identified. G. Separation of the full length of the YY1 3UTR into different overlapping fragments. H. Pull-down assays showed GRSF1 specifically interacted with 3UTR-2205 of YY1. I. Further pull-down assays showed GRSF1 associated with 3UTR 2663-2847 of YY1. J. Luciferase reporter assays suggested GRSF1 interacted with YY1 by binding with 3UTR 2663-2847 of YY1 mRNA. * $p<0.05 \nabla^{* *} p<0.01$. 
A
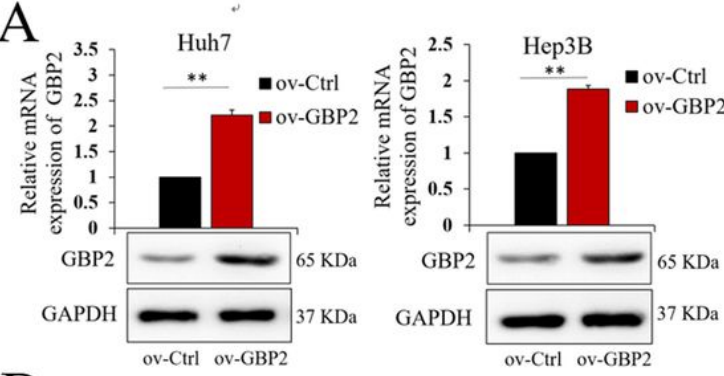

B
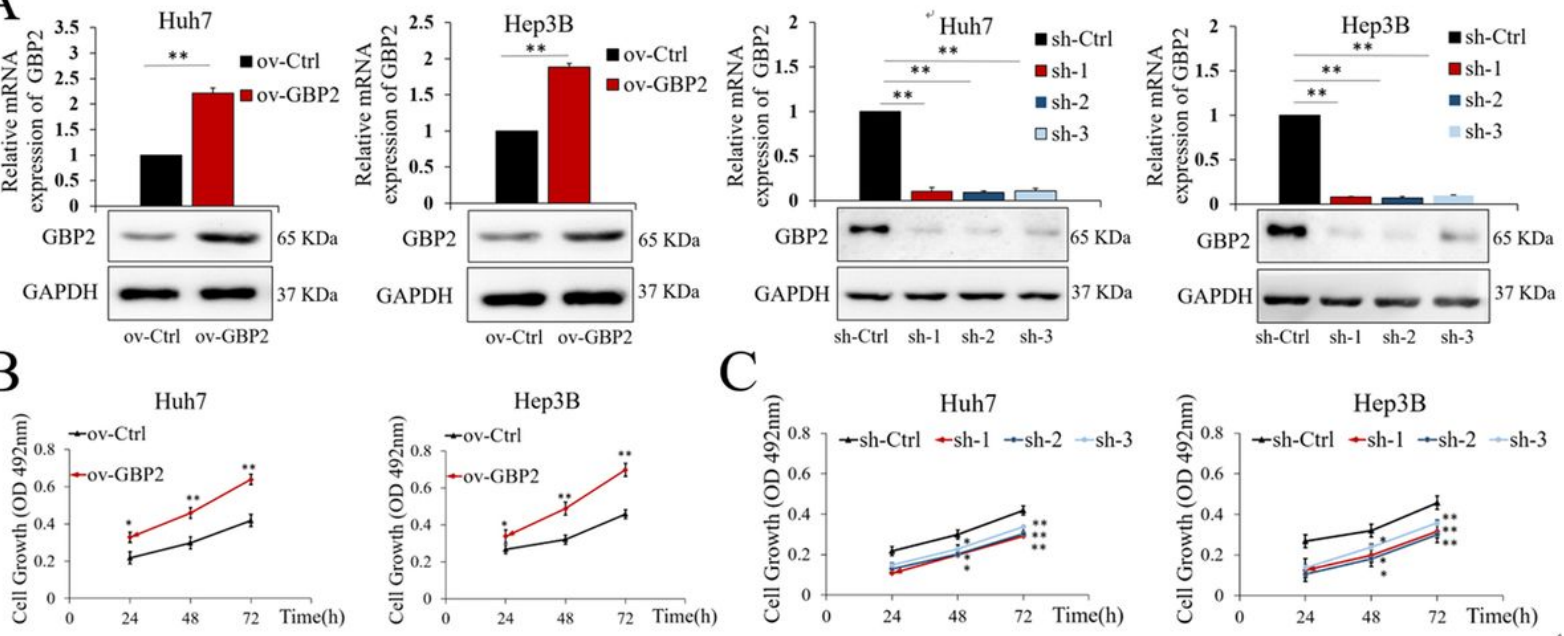

$\mathrm{C}$
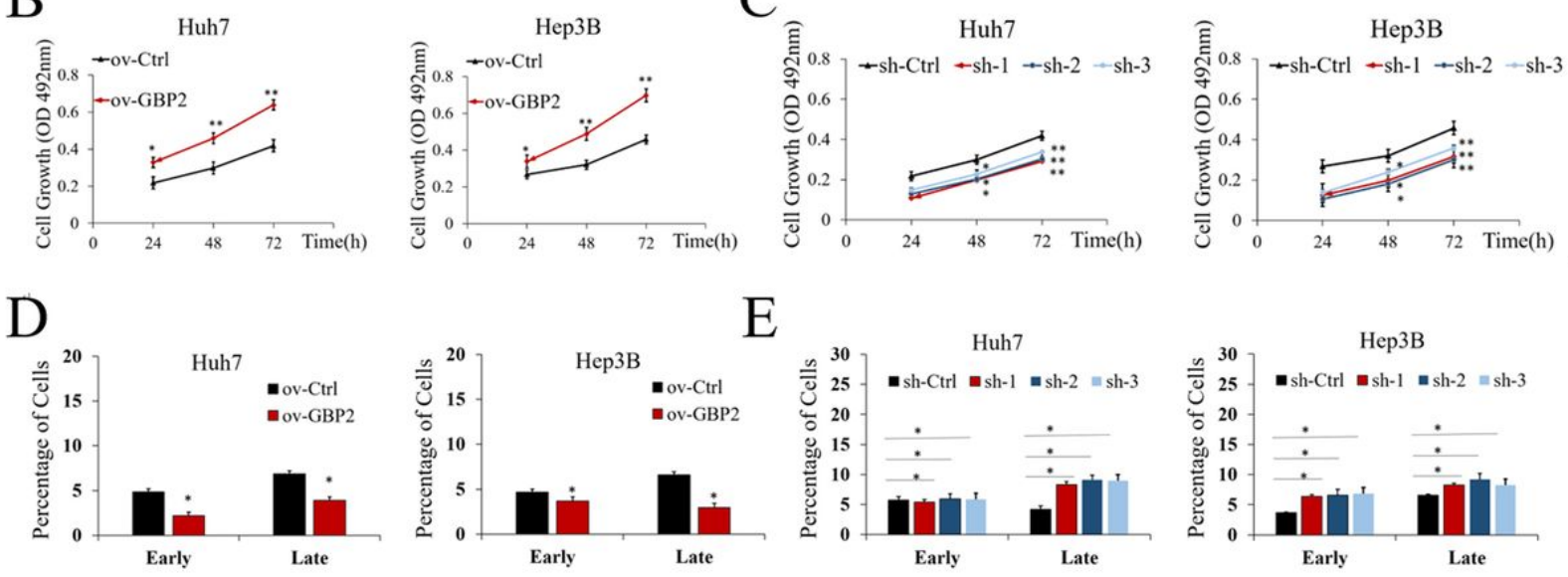

$\mathrm{F}$

\section{$\mathrm{G}$}
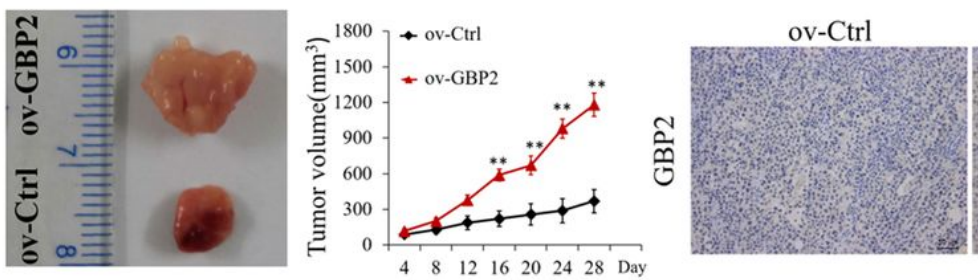

ov-GBP2
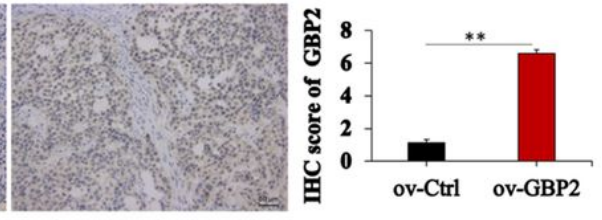

Figure 8

GRSF1 knockdown suppressed HCC malignant biological behaviorinvitro.A. qRT-PCR and western blot were applied to detect GRSF1 mRNA and protein expression levels in MHCC-97H and Hep3B cells transfected with sh-GRSF1 lentivirus (sh-GRSF1-1,sh-GRSF1-2) or negative control lentivirus(sh-Ctrl, respectively. MTT (B), colony formation (C), FCM(D) and Transwell(E) assays showed that GRSF1 knockdown suppressed cells proliferation, colony-forming ability, migration and invasion, while enhance cells apoptosis of MHCC-97H and Hep3B cells. The invaded cells in Transwellassayswere quantified by counting the cells in 10 random fields (magnification, 200x). ${ }^{*} p<0.05 \rrbracket^{\star \star *} p<0.01$. 
A

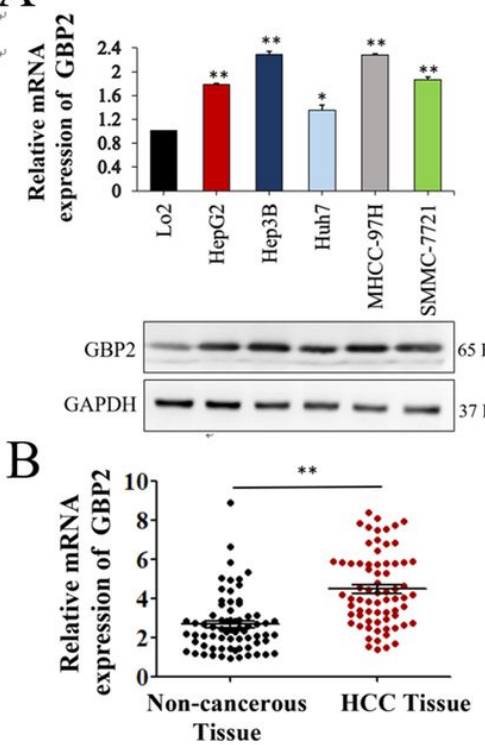

$\mathrm{C}$

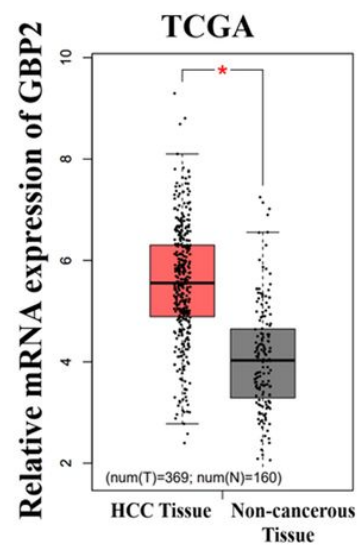

$\mathrm{D}$

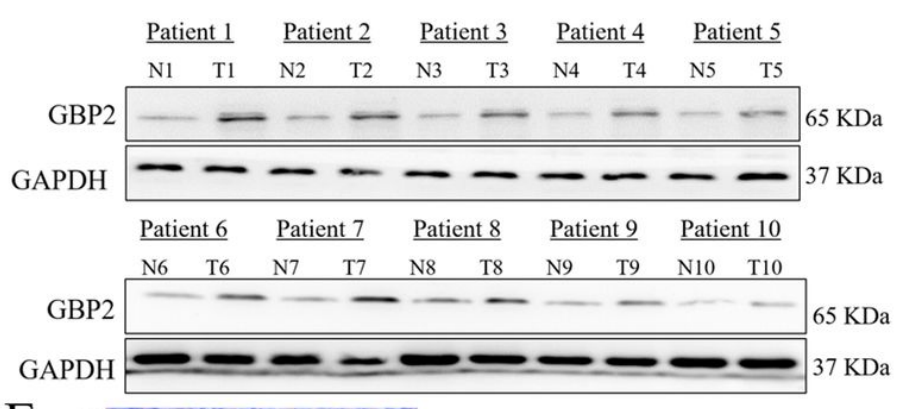

E
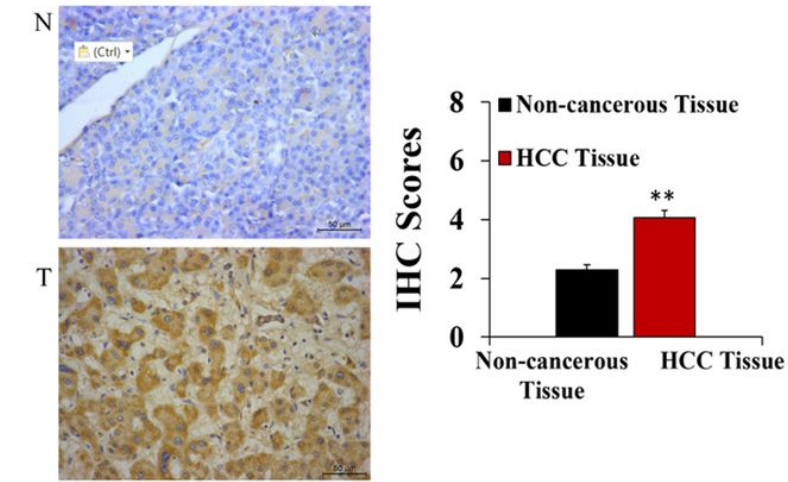

$\mathrm{F}$
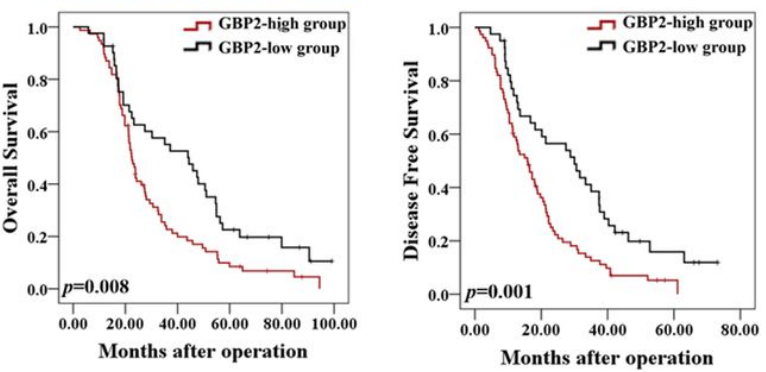

Figure 9

GRSF1 is frequently increased in HCC and is correlated with poor prognosis.A. The analyses of GRSF1 expression in HCC and nontumor tissues in TCGA datasets.B. The correlation between GRSF1 expression levels and prognosisfor HCC patients in TCGAdatasets.C. The analyses of GRSF1 mRNA expression levels in $120 \mathrm{HCC}$ and paired non-cancerous tissues.D.GRSF1 mRNA and protein expression levels in HCC cell lines (Huh7, Hep3B, MHCC-97H, MHCC-97L and Bel-7402)and the immortalized normal human liver cell line (LO2). E. GRSF1 proteinexpression in $10 \mathrm{HCC}$ and paired non-cancerous tissues.F.Kaplan-Meier analysis showing the association between GRSF1 expression andprognosis of 120 HCC patients. ${ }^{*}<<0.05 \rrbracket^{\star *}{ }^{*}<0.01$.

\section{Supplementary Files}

This is a list of supplementary files associated with this preprint. Click to download.

- suppl.docx

- suppl.docx 